\title{
Deciphering cell-cell interactions and communication from gene expression
}

\author{
Erick Armingol $\mathbb{B}^{1,2,3,7}$, Adam Officer $\mathbb{D}^{3,4,7}$, Olivier Harismendy $\mathbb{D}^{4,5} \llbracket$ \\ and Nathan E. Lewis ${ }^{1,2,6 凶}$
}

Abstract | Cell-cell interactions orchestrate organismal development, homeostasis and single-cell functions. When cells do not properly interact or improperly decode molecular messages, disease ensues. Thus, the identification and quantification of intercellular signalling pathways has become a common analysis performed across diverse disciplines. The expansion of protein-protein interaction databases and recent advances in RNA sequencing technologies have enabled routine analyses of intercellular signalling from gene expression measurements of bulk and single-cell data sets. In particular, ligand-receptor pairs can be used to infer intercellular communication from the coordinated expression of their cognate genes. In this Review, we highlight discoveries enabled by analyses of cell-cell interactions from transcriptomic data and review the methods and tools used in this context.

${ }^{1}$ Department of Pediatrics, University of California, San Diego, La Jolla, CA, USA.

${ }^{2}$ Novo Nordisk Foundation Center for Biosustainability at the University of California, San Diego, La Jolla, CA, USA. ${ }^{3}$ Bioinformatics and Systems Biology Graduate Program, University of California, San Diego, La Jolla, CA, USA.

${ }^{4}$ Division of Biomedical Informatics, University of California, San Diego, La Jolla, CA, USA

${ }^{5}$ Moores Cancer Center University of California, San Diego, La Jolla, CA, USA.

${ }^{6}$ Department of Bioengineering, University of California, San Diego, La Jolla, CA, USA.

${ }^{7}$ These authors contributed equally: Erick Armingol, Adam Officer.

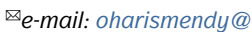
ucsd.edu;nlewisres@ ucsd.edu

https://doi.org/10.1038/ s41576-020-00292-x
Multicellular life relies on the coordination of cellular activities, which depend on cell-cell interactions (CCIs) across an organism's diverse cell types and tissues ${ }^{1-3}$. Thus, studies on cellular functions increasingly require consideration of the community context of each cell ${ }^{4}$. CCIs leverage diverse molecules, including ions, metabolites, integrins, receptors, junction proteins, structural proteins, ligands and secreted proteins of the extracellular matrix. Some molecules support structural CCIs (for example, cell adhesion molecules), whereas ligands such as hormones, growth factors, chemokines, cytokines and neurotransmitters mediate cell-cell communication (CCC) (FIG. 1a). The signalling events behind CCC are often mediated by interactions of various types of protein, encompassing ligand-receptor, receptor-receptor and extracellular matrix-receptor interactions. Receiver cells trigger downstream signalling through cognate receptors, generally culminating in altered transcription factor activity and gene expression. These cells with altered expression further interact with their microenvironment. To understand the role of each cell within its local community, one must identify the protein messages passed between cells; measuring expressed messenger molecules and their associated pathways is fundamental to understanding the directionality, magnitude and biological relevance of CCC.

Direct measurement of proteins mediating CCC requires specialized biochemical assays and extensive domain knowledge; moreover, these proteins cannot always be studied in the native microenvironment. Traditional assays of the underlying protein-protein interactions (PPIs) include yeast two-hybrid screening, co-immunoprecipitation, proximity labelling proteomics, fluorescence resonance energy transfer imaging and X-ray crystallography ${ }^{5,6}$. These techniques have identified many interactions between proteins that are secreted or displayed extracellularly to mediate intercellular communication. Proteomics and transcriptomics can further reinforce such studies as evidence of expression supports the presence of PPIs. This approach has been applied to, for instance, the analysis of communication between 144 human primary cell types, which provided insights into pairs of cells that are more likely to interact and the specific pathways they use to communicate ${ }^{7}$. While proteomic technologies are preferable for these analyses owing to the direct measurement of protein abundances, RNA sequencing (RNA-seq) data sets are more numerous, easier to access and straightforward to analyse. They can also be generated from bulk samples ${ }^{8}$, microdissected specimens ${ }^{9}$ or single-cell suspensions ${ }^{10}$ and enable studies of CCC at different resolutions, whereas proteomics at single-cell resolution is a technology still under development ${ }^{11}$. Single-cell RNA-seq has benefits over bulk analysis, chiefly in quantifying expression in rare cell types and in identifying the cell type of origin of proteins mediating CCIs ${ }^{12,13}$. Results from transcriptomics must be cautiously considered and validated to avoid misleading hypotheses; however, the ubiquity and ease of analysis have enabled many recent studies to infer CCC from gene expression, generating testable hypotheses across diverse disciplines. In particular, the coordinated gene expression of ligands and receptors can be used to infer intercellular communication. 


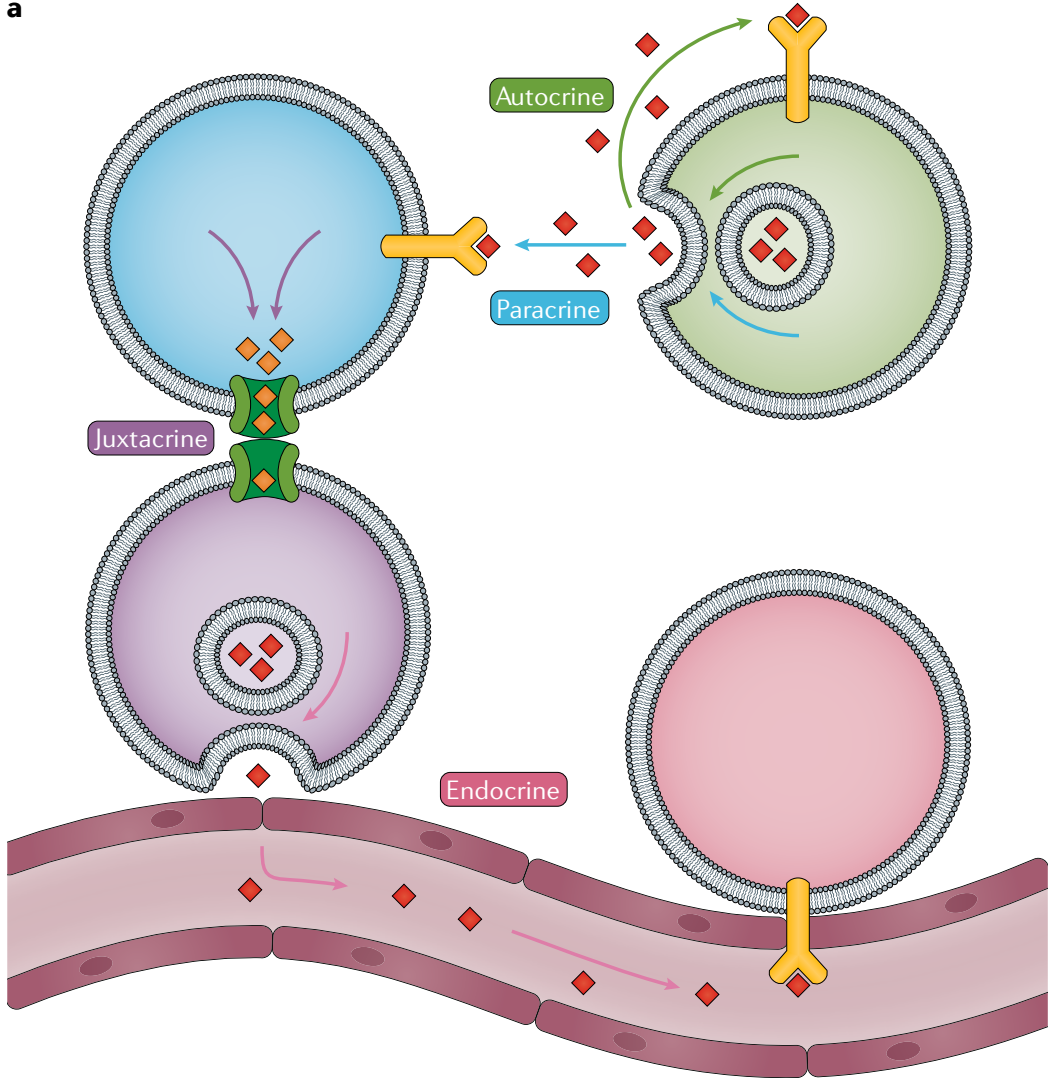

b

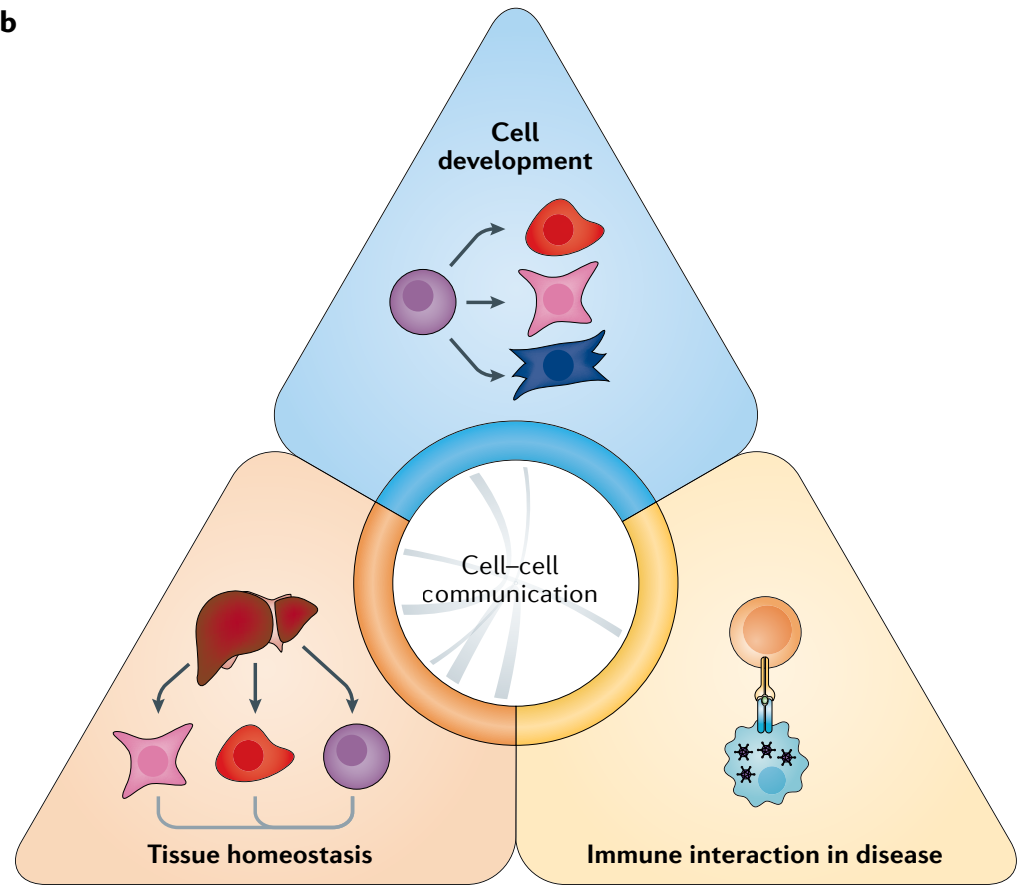

Cell-cell interactions (CCls). Physical interactions between two or more cells, which can be mediated by proteins, ligands, sugars or other biomolecules. of fields that RNA-based CCI analyses have been applied to, illustrating the types of insight that can be gleaned. We then discuss the computational strategies adopted in those studies, detailing the PPI databases and mathematical models commonly used to decipher CCC. Additionally, we introduce the computational tools that
Here, we start by providing an overview of the range
Fig. 1 | Types and applications of cell-cell interactions and communication. a| 'Autocrine signalling' refers to intracellular communication whereby cells secrete ligands that are used to induce a cellular response through cognate receptors for those molecules expressed on the same cell. Paracrine cell-cell communication does not require cell-cell contact, rather depending on the diffusion of signalling molecules from one cell to another after secretion. Juxtacrine, that is, contact-dependent, cellcell communication relies on gap junctions or other structures such as membrane nanotubes to pass signalling molecules directly between cells, without secretion into the extracellular space. Endocrine cell-cell communication represents intercellular communication whereby signalling molecules are secreted and travel long distances through extracellular fluids such as the blood plasma; typical mediators of this communication are hormones.

b | Overview of the main applications of cell-cell interaction methods: cell development, tissue and organ homeostasis, and immune interactions in disease (for more details on each study type, see Supplementary Table 1).

facilitate these analyses, describing their main features as well as their strengths and weaknesses. Finally, we review approaches to validate CCI-derived results and discuss remaining challenges and future directions in the field.

\section{Insights from RNA-based $\mathrm{CCl}$ analyses}

The study of intercellular interactions has greatly accelerated as transcriptomics, in particular bulk and single-cell RNA-seq, has become commonplace. These approaches use transcriptional profiling to decipher CCCs at any stage of development and in any multicellular community. Many studies focus on signals mediating cellular differentiation, interactions of cell types within tissues and organs, and immune responses (FIG. 1 b; TABLE 1; see Supplementary Table 1 for more details). Here, we review these studies and illustrate the types of insights gained from analysing CCC.

Interactions drive cellular differentiation and organ development. Cellular differentiation depends on temporally and locally precise cell communication, so inspecting intercellular communication has increased our understanding of stem cell fates and revealed ligand-receptor interactions that initiate self-renewal and differentiation ${ }^{14-20}$. For example, a CCC network of haematopoietic cells built using ligand-receptor pairs showed that fate decisions are regulated through precise coordination of an antagonistic feedback circuit involving megakaryocyte-derived stimulatory factors and monocyte-derived inhibitory factors ${ }^{14}$. Another analysis of CCC networks interrogating how differentiated cells influence haematopoietic stem cell fate revealed that ligand production is cell type specific, whereby some cells can produce signals with the same function, whereas receptors are less specific ${ }^{15}$. Given the promiscuity of receptors, physical compartmentalization of cells is key to limit ligand signalling and confer specificity to stem cell fates.

Tissue and organ development also depends on signals that progenitor cells send and receive ${ }^{21}$. The analysis of brain CCC showed crosstalk involved in neurogenesis and identified novel mediators ${ }^{16,22}$, such 
Table 1 | Illustrative studies and their strategies for deciphering cell-cell interactions and communication

\begin{tabular}{|c|c|c|c|c|c|c|c|}
\hline Sample or organ & Key input & Scoring & CS value & CCC score & Validation & Study focus & Ref \\
\hline \multicolumn{8}{|l|}{ Cell development } \\
\hline $\begin{array}{l}\text { Haematopoietic } \\
\text { cells (human) }\end{array}$ & Microarray; LRIs & $\begin{array}{l}\text { Expression } \\
\text { thresholding }\end{array}$ & Binary & No score & $\begin{array}{l}\text { Functional } \\
\text { validation }\end{array}$ & $\begin{array}{l}\text { Role of CCC between } \\
\text { differentiated haematopoietic } \\
\text { cells and HSCs in fate decisions }\end{array}$ & 15 \\
\hline $\begin{array}{l}\text { Brain (mouse } \\
\text { embryonic cortex) }\end{array}$ & Microarray; LRIs & $\begin{array}{l}\text { Expression } \\
\text { thresholding }\end{array}$ & Binary & No score & $\begin{array}{l}\text { Functional } \\
\text { validation }\end{array}$ & $\begin{array}{l}\text { Role of microenvironment } \\
\text { in self-renewal versus } \\
\text { differentiation decision of } \\
\text { neural precursor cells during } \\
\text { neurogenesis }\end{array}$ & 16 \\
\hline $\begin{array}{l}\text { Liver and iPS cells } \\
\text { (human) }\end{array}$ & scRNA-seq; LRIs & $\begin{array}{l}\text { Expression } \\
\text { thresholding }\end{array}$ & Binary & $\begin{array}{l}\text { Normalized } \\
\text { sum of CS }\end{array}$ & $\begin{array}{l}\text { Functional } \\
\text { validation }\end{array}$ & $\begin{array}{l}\text { 3D liver bud organoid from } \\
\text { iPS cells to characterize CCC } \\
\text { shaping hepatogenesis }\end{array}$ & 24 \\
\hline Placenta (human) & scRNA-seq; LRIs & $\begin{array}{l}\text { Expression } \\
\text { thresholding }\end{array}$ & Binary & No score & $\begin{array}{l}\text { Functional } \\
\text { validation }\end{array}$ & $\begin{array}{l}\mathrm{CCl} \text { in the fetus-placenta } \\
\text { interface before and after } \\
\text { decidualization }\end{array}$ & 29 \\
\hline ¡PS cells (mouse) & scRNA-seq; LRIs & Expression product & Continuous & Sum of CS & $\begin{array}{l}\text { Functional } \\
\text { validation }\end{array}$ & $\begin{array}{l}\mathrm{CCC} \text { at the beginning of } \\
\text { differentiation }\end{array}$ & 19 \\
\hline $\begin{array}{l}\text { Bone marrow } \\
\text { (mouse) }\end{array}$ & scRNA-seq; LRIs & RNA-Magnet & Continuous & No score & Colocalization & $\begin{array}{l}\text { CCC and interactions between } \\
\text { bone marrow cells }\end{array}$ & 53 \\
\hline \multicolumn{8}{|l|}{ Tissue interactions } \\
\hline $\begin{array}{l}\text { Multiple lineages } \\
\text { (human) }\end{array}$ & scRNA-seq; LRIs & $\begin{array}{l}\text { Expression } \\
\text { thresholding }\end{array}$ & Binary & Sum of CS & None & $\begin{array}{l}\text { CCC between multiple cell } \\
\text { lineages }\end{array}$ & 7 \\
\hline Lungs (human) & popRNA-seq; LRIs & $\begin{array}{l}\text { Expression } \\
\text { thresholding }\end{array}$ & Binary & Sum of CS & $\begin{array}{l}\text { Colocalization } \\
\text { functional } \\
\text { validation }\end{array}$ & $\begin{array}{l}\text { Signals sent by mesenchymal } \\
\text { cells in lungs that are key } \\
\text { for self-renewal of epithelial } \\
\text { progenitors after tissue injury }\end{array}$ & 40 \\
\hline $\begin{array}{l}\text { Immune system } \\
\text { and structural } \\
\text { cells (mouse) }\end{array}$ & $\begin{array}{l}\text { Low-input } \\
\text { RNA-seq; LRIs }\end{array}$ & $\begin{array}{l}\text { Differential } \\
\text { combinations }\end{array}$ & Binary & Odds ratios & $\begin{array}{l}\text { Functional } \\
\text { validation }\end{array}$ & $\begin{array}{l}\text { Role of structural cells in } \\
\text { immune responses }\end{array}$ & 58 \\
\hline Heart (mouse) & scRNA-seq; LRIs & $\begin{array}{l}\text { Differential } \\
\text { combinations }\end{array}$ & Binary & Sum of CS & $\begin{array}{l}\text { Expression; } \\
\text { colocalization } \\
\text { functional } \\
\text { validation }\end{array}$ & $\begin{array}{l}\text { CCC of cardiomyocytes and } \\
\text { non-cardiomyocytes in human } \\
\text { heart in health and under } \\
\text { failure }\end{array}$ & 34 \\
\hline Placenta (human) & scRNA-seq; LRIs & CellPhoneDB & Continuous & No score & Colocalization & $\begin{array}{l}\text { Key ligand-receptor pairs } \\
\text { based on subunit architecture; } \\
\text { CCC at maternal-fetal interface }\end{array}$ & 30 \\
\hline \multicolumn{8}{|c|}{ Tumour microenvironment } \\
\hline $\begin{array}{l}\text { Melanoma } \\
\text { (human) }\end{array}$ & scRNA-seq; LRIs & $\begin{array}{l}\text { Expression } \\
\text { thresholding }\end{array}$ & Binary & Sum of CS & None & $\mathrm{CCl}$ network of isolated cells & 70 \\
\hline HNSCC (human) & scRNA-seq; LRIs & $\begin{array}{l}\text { Expression } \\
\text { thresholding }\end{array}$ & Binary & No score & Colocalization & $\begin{array}{l}\text { CCC in patients with HNSCC } \\
\text { generated by HPV or } \\
\text { environmental carcinogens } \\
\text { (HPV negative) }\end{array}$ & 61 \\
\hline $\begin{array}{l}\text { Five cancer types } \\
\text { (mouse) }\end{array}$ & scRNA-seq; LRIs & Expression product & Continuous & No score & None & $\begin{array}{l}\mathrm{CCC} \text { within a tumoural } \\
\text { microenvironment }\end{array}$ & 60 \\
\hline $\begin{array}{l}\text { Nine cancer types } \\
\text { (human) }\end{array}$ & Microarray; LRIs & $\begin{array}{l}\text { Expression } \\
\text { correlation (Pearson) }\end{array}$ & Continuous & No score & None & $\begin{array}{l}\text { Correlation between autocrine } \\
\text { signalling pathways and } \\
\text { mRNA levels of ligands } \\
\text { and receptors }\end{array}$ & 69 \\
\hline
\end{tabular}


Table 1 (cont.) | Illustrative studies and their strategies for deciphering cell-cell interactions and communication

\begin{tabular}{|c|c|c|c|c|c|c|c|}
\hline Sample or organ & Key input & Scoring & CS value & CCC score & Validation & Study focus & Ref \\
\hline \multicolumn{8}{|c|}{ Tumour microenvironment (cont.) } \\
\hline Lungs (human) & scRNA-seq; LRIs & $\begin{array}{l}\text { Differential } \\
\text { combination; } \\
\text { expression } \\
\text { thresholding }\end{array}$ & Binary & No score & $\begin{array}{l}\text { Functional } \\
\text { validation }\end{array}$ & $\begin{array}{l}\text { Tumour-stroma CCC in } \\
\text { lung cancer; introduced } \\
\text { CCCExplorer }\end{array}$ & 67 \\
\hline Ovary (human) & $\begin{array}{l}\text { Microarray; LRIs; } \\
\text { downstream } \\
\text { target genes }\end{array}$ & $\begin{array}{l}\text { Differential } \\
\text { combination; } \\
\text { expression } \\
\text { thresholding }\end{array}$ & Binary & No score & $\begin{array}{l}\text { Expression; } \\
\text { functional } \\
\text { validation }\end{array}$ & $\begin{array}{l}\text { CCC between stromal and } \\
\text { ovarian cancer cells }\end{array}$ & 68 \\
\hline $\begin{array}{l}\text { Head and neck } \\
\text { and immune } \\
\text { system (human) }\end{array}$ & $\begin{array}{l}\text { scRNA-seq; LRIs; } \\
\text { downstream } \\
\text { target genes }\end{array}$ & NicheNet & Continuous & No score & None & $\begin{array}{l}\text { Prediction of ligand-target } \\
\text { links between interacting cells; } \\
\text { tested on HNSCC data set }\end{array}$ & 54 \\
\hline
\end{tabular}

\section{Receptors}

Proteins that bind to other biomolecules to receive or amplify a signal. They are most commonly membrane-bound but can also be found in the cytoplasm.

Ligands

Biomolecules that bind to receptors and change the activity, conformation or other biological properties of the receptor, triggering a signalling event.

Extracellular matrix

Three-dimensional organization of biomolecules located in the extracellular space. It provides structural and functional

support to neighbouring cells.

Cell-cell communication

(CCC). Subset of cell-cell

interactions involving

biochemical signals that

are sent between or within

cells and generate an

intracellular effect.

Protein-protein interactions (PPIs). Physical interaction between two proteins, often involved in structural systems, signal transduction or metabolic processes.

Network

A set of nodes with defined pairwise attributes. For

example, a protein-protein interaction network would

consist of proteins as nodes with attributes linking nodes that are known to interact with each other. as apolipoprotein E (APOE), a protein associated with Alzheimer disease. CCC analysis also elucidated how erythroblasts interact with macrophages during haematopoiesis in the fetal liver ${ }^{23}$ and was applied to liver organoid development to investigate how multilineage communication shapes the differentiation of hepatic cells $^{24}$. Following similar principles, other studies have used CCC analyses to understand differentiation during epidermal regeneration ${ }^{25-27}$, development of the interfollicular epidermis ${ }^{28}$ and formation of the maternal-fetal interface in early pregnancy ${ }^{29,30}$.

Cell-type communication in tissue and organ homeostasis. Expression profiling of different cell types in adult tissues and organs has shown how intercellular communication contributes to organ function. To date, this approach has been applied to brain ${ }^{31,32}$, heart ${ }^{33,34}$, kidney $^{35-37}$, liver ${ }^{38,39}$, lungs ${ }^{40-43}$, placenta ${ }^{29,30,44}$, retina ${ }^{45}$ and visual cortex ${ }^{46}$. Remarkably, this approach revealed new roles of cells within a tissue ${ }^{41}$ and helped explain how ageing $^{32,47}$, diseases ${ }^{34-36,48-51}$, infections ${ }^{52}$ and injuries ${ }^{31,40,43}$ shape multicellular organization. Intercellular crosstalk has been investigated in healthy and diseased liver ${ }^{50}$, kidneys during homeostasis and rejected kidney transplants ${ }^{35,36}$, heart during failure and recovery conditions $^{34}$, and healthy and asthmatic lungs ${ }^{51}$. RNAbased strategies for studying CCIs and CCC have helped elucidate, for instance, how cells communicate during ageing of the mouse brain. This revealed that CST3 and CXCL12 are mediators that differentiate intercellular interactions in young and old brains and may modulate ageing-related processes ${ }^{32}$. Thus, within-tissue CCC studies continue to deepen our understanding, for basic and therapeutic purposes, of how cellular communities work.

During the study of CCC, considering spatial context clarifies relationships between cells across tissues and organs. Several studies have incorporated imaging to spatially map cells and integrated transcriptomics to measure CCC. For example, researchers interrogated the communication and response of lung cells to tissue injury ${ }^{40,43}$; spatial information aided the identification of a new population of lung endothelial cells (Car4 high) and elucidated how these cells communicate with neighbouring alveolar type 1 cells through
VEGF signalling given both their proximity and their expression of the cognate genes ${ }^{43}$. Spatial maps have also helped investigate the communication of $\mathrm{T}$ cells during their development in the human thymus ${ }^{13}$. As this process is spatially coordinated, knowing the cellular localizations was crucial to understand, for example, interactions between XCR $1^{+}$dendritic cells and T cells with high expression of XCL1, which are important to recruit dendritic cells into the medulla of the thymus. Yet another study inferred the 3D organization of bone marrow - instead of using tissue imaging to map the cells - ultimately identifying signalling between immune and non-immune cells ${ }^{53}$. These examples show how cell localization can help elucidate interactions between spatially proximal regions.

Immune interactions in disease. The immune system receives signals from multiple tissues, but only specific signals allow it to coordinate healthy immune responses. For instance, CCL2- and $\mathrm{CX}_{3} \mathrm{CL} 1$-mediated communication coordinate the recruitment and positioning of immune cells, as determined from singlecell transcriptomes ${ }^{37,39}$. Specifically, these CCCs were associated with the recruitment of monocytes that later became liver-resident macrophages ${ }^{39}$ and the positioning of mononuclear phagocytes in kidney ${ }^{37}$, which are crucial processes to combat ascending uropathogenic Escherichia coli infection. CCC is also involved in the response to viral infections ${ }^{52,54-56}$. Studies of respiratory diseases investigated the crosstalk between lung and $\mathrm{T}$ cells in Sendai virus-infected mice ${ }^{55}$ or CCC associated with severe acute respiratory syndrome coronavirus 2 (SARS-CoV-2) infection ${ }^{52,56}$, where interactions between immune and epithelial cells correlated with COVID-19 severity. CCC-based studies have also provided more general insights. For example, they helped build a social network of immune cells by identifying communication pathways between immune cells ${ }^{57}$. Furthermore, they revealed the roles of structural cells in immune responses by elucidating how fibroblasts, endothelial cells and epithelial cells are primed for organ-specific immune gene activation through upregulation of ligands and receptors, including $\beta_{2} \mathrm{M}, \mathrm{CD} 74$, CXCL10, VCAM1 and TNFRSF1A ${ }^{58}$. 
Tumours and their surrounding microenvironments are complex communities of cells that modify local immune cell functions. Studying CCC within these communities can reveal how cells communicate in these ecosystems ${ }^{54,59-64}$ and help guide the development of effective cancer immunotherapies just as the inhibition of CCC through PD1 and PDL1 has revolutionized the field ${ }^{65,66}$. CCC analyses have also elucidated crosstalk between tumour and stroma ${ }^{67,68}$ and communication pathways used by tumours ${ }^{6-71}$. Several studies have developed statistical models to connect inferred CCC mechanisms to cancer phenotypes. One case observed clear correlations between the activity of specific ligandreceptor interactions and the degree of regulatory $\mathrm{T}$ cell infiltration and tumour growth ${ }^{60}$. In another study, active ligand-receptor pairs were associated with invasiveness and proliferation of malignant cells under a partial epithelial-mesenchymal transition programme in patients with head and neck squamous cell carcinoma ${ }^{59}$. Moreover, the expression levels of key mediators of CCC were used as inputs for training a decision tree to predict prognosis for patients with glioma ${ }^{71}$; this model classified patients into high-risk and low-risk groups, defined on the basis of the difference in patient survival time. Thus, studying CCC within the tumour microenvironment provides opportunities to identify druggable pathways and develop new cancer therapeutics ${ }^{68}$.

\section{Deciphering CCC}

The aforementioned studies provide a glimpse of the insights attainable when studying CCC. While the methods and tools that these studies used have in common that they infer CCC from gene expression (FIG. 2a), a diverse range of strategies can be applied (FIG. 2b; TABLE 1). For simplicity, we refer to them as methods for studying CCC, but these strategies can decipher any type of gene product-based CCIs, including proteins that participate in structural interactions between cells. Furthermore, although we focus on mammalian CCIs, the approaches apply to any prokaryotic or eukaryotic cells with a characterized interactome (for example, Drosophila melanogaster ${ }^{72,73}$ and Caenorhabditis elegans $^{74,75}$ ).

Building from PPIs. Inferring CCC from transcriptomics relies on gene co-expression, whereby one gene in a given pair comes from one interacting cell and the other gene comes from the second interacting cell. Several studies focused on intercellular signalling using co-expression of all genes or specific cell markers ${ }^{31,76}$, the similarity between expression profiles ${ }^{77}$ or the properties of regulatory networks ${ }^{78}$. However, most studies rely on literature-curated lists of interacting proteins, which facilitates the biological interpretation of results (FIG. 2a). Although several studies have used interactions between any class of cell-surface protein and secreted protein $^{32,53}$, the predominant class of interactions used for studying CCC are known ligands and their cognate receptors.

By focusing on ligands and receptors, an early study investigated autocrine signalling loops in cancer ${ }^{69}$. The authors analysed the correlation between ligand expression and cognate receptor expression. They first created a literature-curated list of ligand-receptor pairs (Database of Ligand-Receptor Partners), which was subsequently integrated with microarray-based expression data to analyse autocrine signalling in cancer cells. At the time, the Database of Ligand-Receptor Partners consisted of 451 interactions in humans; since then, many other databases have catalogued substantially more interacting ligand-receptor pairs, enabling more comprehensive testing of communication processes between cells.

Many studies have enumerated known ligand-receptor pairs using different approaches (BOX 1). To facilitate further use and comparison, we collated publicly available lists into a single ligand-receptor pair repository. One extensively used database $\mathrm{e}^{7}$ contains $\sim 2,400$ human ligand-receptor pairs, obtained from multiple databases and literature curation. Similar approaches allowed researchers to increase the number of known ligand-receptor pairs and to build databases for other organisms (Supplementary Table 1). However, integrating multiple sources of data is challenging and requires reconciliation of the different ways ligand-receptor pair confidence was assessed or how orthologues were determined. Furthermore, few predicted PPIs in databases have been validated, raising the concern of false positives.

While early efforts increased the number of reported ligand-receptor pairs, many lacked information about protein complexes involving multiple subunits. This scenario greatly increases false-positive CCC predictions ${ }^{79}$. Some proteins, such as transforming growth factor- $\beta$ receptors ${ }^{80}$ and cytokine receptors ${ }^{81}$, require multisubunit assembly for function. A lack of expression of any subunit blocks ligand-receptor interactions and the resulting communication ${ }^{82}$. Thus, more recent computational tools such as CellPhoneDB ${ }^{30,83}$, CellChat ${ }^{79}$ and ICELLNET $^{84}$ include multimeric proteins and interactions between complexes of both ligands and receptors. Accounting for subunit co-expression better represents functional ligand-receptor interactions. For example, CellChat $^{79}$ includes $~ 2,000$ ligand-receptor interactions, $\sim 48 \%$ of which represent interactions of heterodimers, a substantial increase from the $\sim 900$ ligand-receptor pairs in CellPhoneDB.

Additional efforts have incorporated information beyond ligand-receptor pairs to reveal other aspects of CCC, such as the interchange of metabolites or activation of intracellular signalling. For example, metabolite secretion data have been included in communication studies by considering the expression of the producing enzymes $^{85}$. In this regard, the production of specific metabolites can be inferred from transcriptomic data ${ }^{86,87}$; however, metabolite concentrations cannot be directly predicted, so concentration-dependent interactions cannot be assessed. Among other efforts, information about downstream signalling gene products and gene regulatory networks can be included ${ }^{25,54}$ by weighing the potential of using a communication pathway given the intracellular effect on a receiver cell. For this, genes in a downstream pathway to each receptor are obtained from signalling and regulatory networks; then their expression provides additional inputs for scoring the respective ligand-receptor pairs. However, more extensive 
1 Collection of samples or cells for transcriptomic analysis

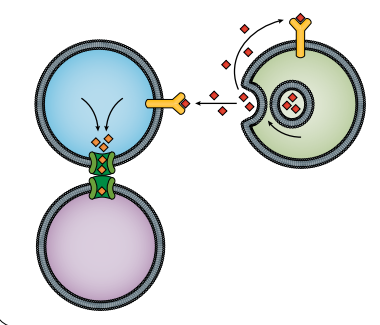

2 Data preprocessing and generation of expression matrix

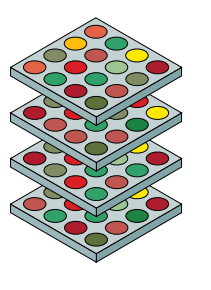

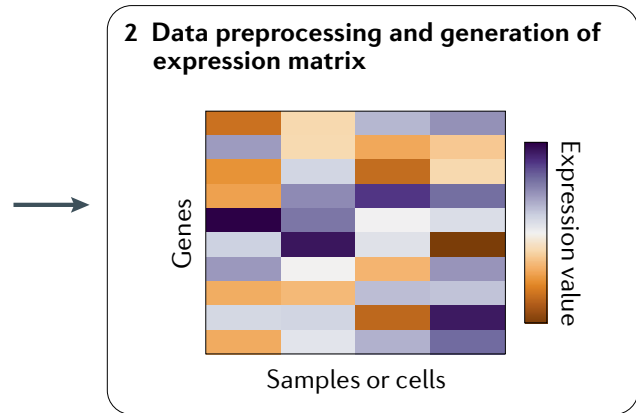

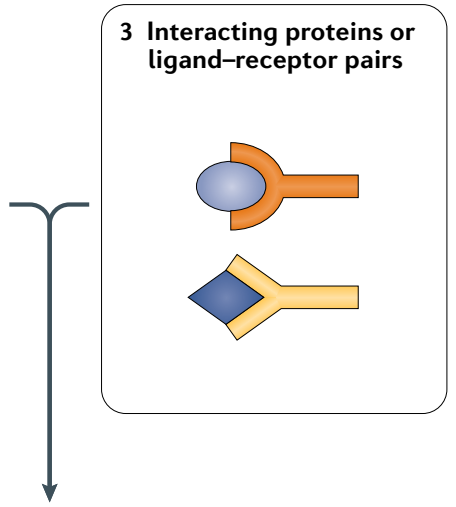

5 Cell-cell interactions and communication analysis

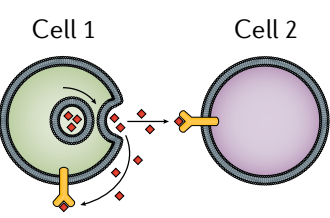

Ligands (cell 1) Receptors (cell 2)

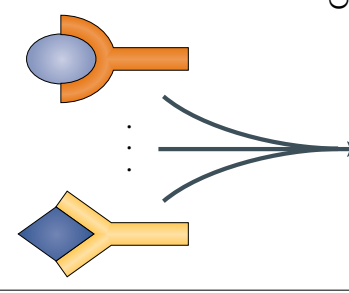

Samples or cells
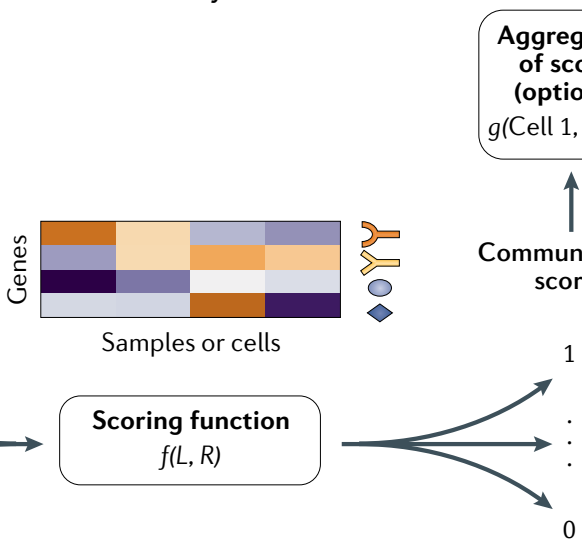

4 Filtering by interacting proteins or ligand-receptor pairs

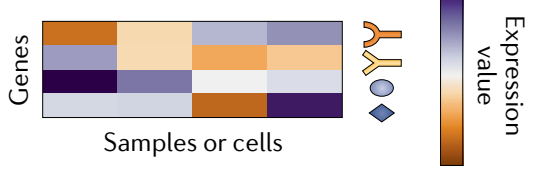

6 Interpretation and visualization of results

Sample

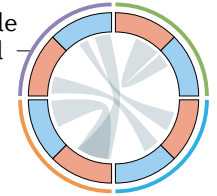

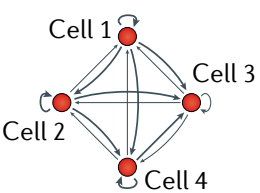

b

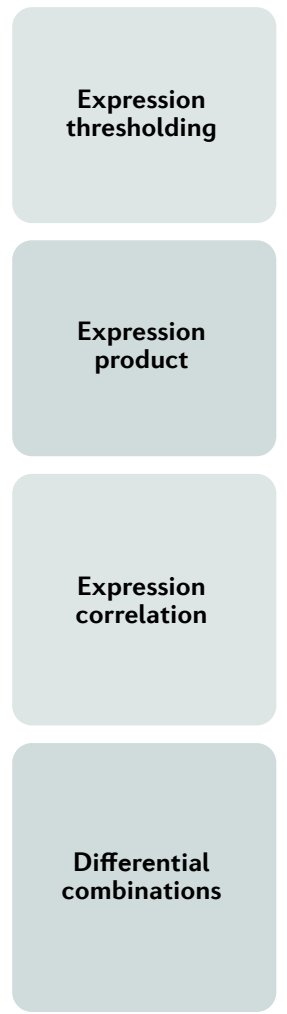

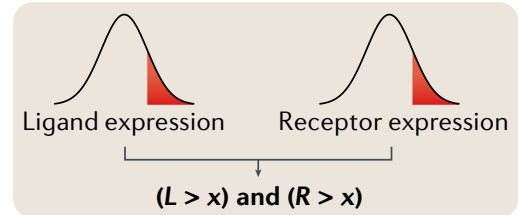
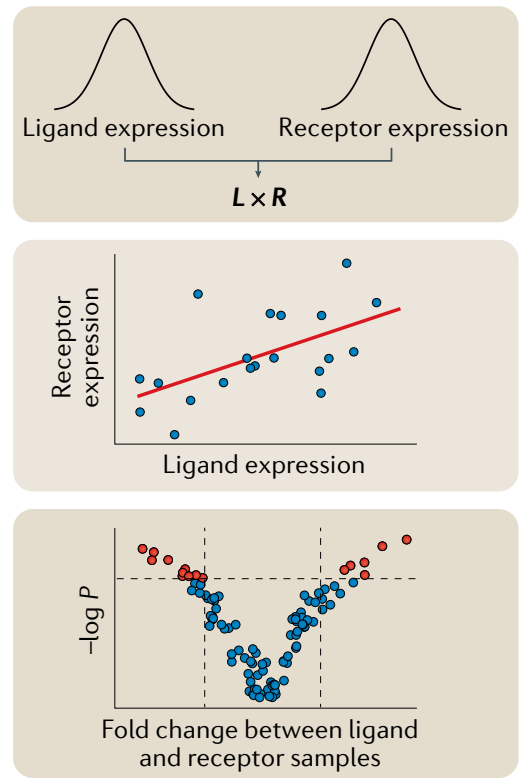

Recommended data

Communication score

Bulk, single cell

Binary

Single cell

Continuous

Bulk, single cell

Continuous 
Fig. 2 | Analysis workflow for inferring cell-cell interactions and communication from gene expression. a | Samples or cells are analysed by transcriptomics to measure the expression of genes (step 1). Then the data generated are preprocessed to build a gene expression matrix, which contains the transcript levels of each gene across different samples or cells (step 2). A list of interacting proteins that are involved in intercellular communication is generated or obtained from other sources (step 3), often including interactions between secreted and membrane-bound proteins (commonly ligands and receptors, respectively). Only the genes associated with the interacting proteins are held in the gene expression matrix (step 4). Their expression levels are used as inputs to compute a communication score for each ligand-receptor pair using a scoring function (function $f(L, R)$, where $L$ and $R$ are the expression values of the ligand and the receptor, respectively). These communication scores may be aggregated to compute an overall state of interaction between the respective samples or cells using an aggregation function (function $g$ (Cell 1, Cell 2), where Cell 1 and Cell 2 are all communication scores of those cells or corresponding samples) (step 5). Finally, communication and aggregated scores can be represented by, for instance, Circos plots and network visualizations to facilitate the interpretation of the results (step 6). $\mathbf{b}$ | Main scoring functions of communication pathways based on the expression of their components. Recommended data to use with these functions and the type of their resulting communication score are indicated.

False negatives

A false negative occurs when a positive example is assigned

a negative label. For the purpose of this Review, this means a true interacting ligand-receptor pair is labelled as not interacting

Post-translational modifications

Covalent modification of amino acid residues on a protein, commonly altering function, structure or localization. Phosphorylation, acetylation and glycosylation are among the most common.

Signalling pathways The network of biomolecules that serve to transmit signals and induce cellular responses. Post-translational modification of proteins is the most common way signals are propagated. information on ligand-receptor pairs is required to include downstream genes. A limitation of this information is that rules of gene regulation are not considered, leading to potential false positives and false negatives.

Adding information can be laborious, requiring careful curation of mediator molecules and development of algorithms that incorporate these data. Furthermore, inference methods based on PPI databases are sensitive to the quality of the underlying databases. Nevertheless, PPIs, especially ligand-receptor pairs, have been central to decipher CCC in all strategies.

Which communication pathways are cells using? To elucidate which biological processes are used by interacting cells to communicate, a score is usually estimated for each pair of interacting proteins (BOX 1) using the expression of their cognate genes as input to a scoring function (FIG. 2a). Importantly, the main assumption of these methods is that (1) gene expression reflects protein abundance and (2) protein abundance is sufficient to infer the PPI strength, ignoring essential factors for their binding, such as post-translational modifications or multisubunit complex assembly. Here, we focus on ligand-receptor pairs as PPIs (FIG. 2a, step 3); however, these strategies can be extended to any intercellular PPIs.

Communication scores can be binary or continuous (FIG. 2b), each providing different insights into the signalling pathways that cells use. Binary scores are simpler, whereas continuous scores enable more precise quantification of intercellular signalling. For both types of communication scores, we identified two core scoring functions in the literature. The 'expression thresholding' and 'differential combinations' methods are defined for the binary category, whereas the 'expression product' and 'expression correlation' approaches quantify continuous scores.

In binary scoring, expression thresholding is widely used because of its easy implementation and interpretation (TABLE 1). By thresholding expression values of both interacting partners in each ligand-receptor pair, we can measure all communication processes used between cells. If both genes are expressed above a threshold, the ligand-receptor pair is considered 'active'; otherwise it is 'inactive' (assigning ones and zeros, respectively; FIG. 3). Different thresholds can be used for the ligand and receptor ${ }^{44,67}$. By contrast, the differential combinations method uses any approach to quantify differential gene expression ${ }^{88,89}$ to identify 'active' interacting partners. Thus, this strategy measures communication pathways in a sample- or cell type-specific manner. Regarding input data sets, both binary functions are suitable for either bulk or single-cell data (FIG. 2b); however, bulk-derived samples may miss certain interacting ligand-receptor pairs, as averaging gene expression across all cells can mask signals from low-abundance cells. These binary methods both assume that higher expression is needed for interaction and require the choosing of a gene expression threshold (either significance or gene expression magnitude), which can lead to false positives and/or false negatives ${ }^{7}$. General thresholds may fail as (1) some proteins have different bioactivities in a concentration-dependent manner ${ }^{90}$ and (2) mRNAprotein level correlations vary across genes ${ }^{82}$. Hence, gene-specific thresholds should be developed, as a general threshold may not properly represent the presence and activity of proteins.

Modelling interactions as non-binary processes provides biologically relevant information ${ }^{91}$ and helps infer active communication pathways. The expression product method computes a continuous value by multiplying the expression of both interacting proteins, and it has successfully found differences in ligand-receptor pair use between cell ${ }^{55,60}$. For example, communication pathways that were previously described to enhance the expansion of regulatory $\mathrm{T}$ cells were also identified to have communication scores linearly correlated with the infiltration of those cells in human metastatic melanoma ${ }^{60}$, which would not have been possible with binary scores. However, this approach may become problematic if interacting proteins have vastly different transcript levels wherein one protein dominates the interaction signal. Additional data preprocessing, such as cell-type normalization of gene expression using housekeeping genes ${ }^{60}$ or accounting for correlation between transcripts and proteins ${ }^{7}$, may mitigate this effect. Although this method can be applied to bulk data, microdissected or single-cell data sets are preferred as they capture the full heterogeneity of expression across cells ${ }^{19,48,49,55,60,62}$, resulting in clearer differences in expression ${ }^{92}$.

The second continuous scoring function is the expression correlation method. This score is the correlation between expression values of the interacting proteins across many samples. Thus, the score represents the general behaviour of each ligand-receptor pair in the evaluated groups rather than the individual importance for each pair of samples or cells. Whereas the previous methods can use any gene expression data type, the expression correlation method requires data sets containing many measurements of two populations to compute correlation for each ligand-receptor pair. A potential obstacle for this method is the sparsity of single-cell data sets, which can increase or decrease correlation coefficients in undesirable ways ${ }^{93,94}$, leading to correlation values that measure sparsity, rather than biology. In addition, the formation of subgroups of data 


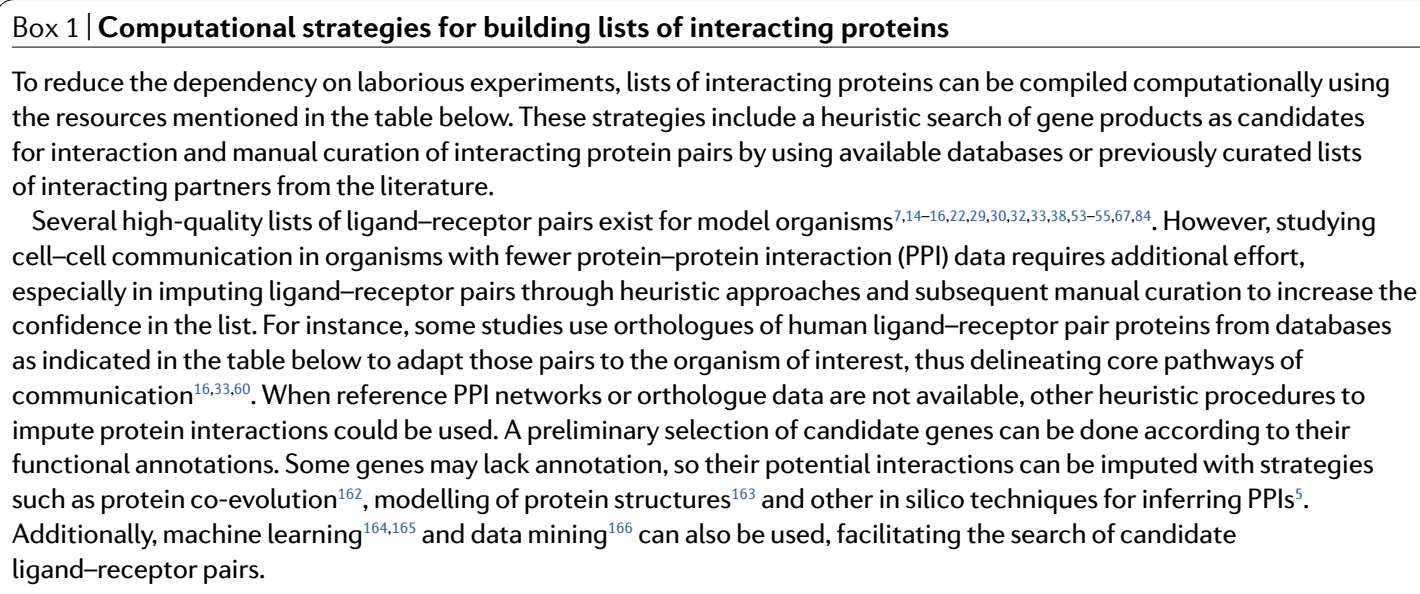

To reduce the dependency on laborious experiments, lists of interacting proteins can be compiled computationally using the resources mentioned in the table below. These strategies include a heuristic search of gene products as candidates for interaction and manual curation of interacting protein pairs by using available databases or previously curated lists of interacting partners from the literature.

Several high-quality lists of ligand-receptor pairs exist for model organisms s $^{7,14-16,22,29,30,32,33,38,53-55,67,84}$. However, studying cell-cell communication in organisms with fewer protein-protein interaction (PPI) data requires additional effort, especially in imputing ligand-receptor pairs through heuristic approaches and subsequent manual curation to increase the confidence in the list. For instance, some studies use orthologues of human ligand-receptor pair proteins from databases as indicated in the table below to adapt those pairs to the organism of interest, thus delineating core pathways of communication ${ }^{16,33,60}$. When reference PPI networks or orthologue data are not available, other heuristic procedures to impute protein interactions could be used. A preliminary selection of candidate genes can be done according to their functional annotations. Some genes may lack annotation, so their potential interactions can be imputed with strategies such as protein co-evolution ${ }^{162}$, modelling of protein structures ${ }^{163}$ and other in silico techniques for inferring $\mathrm{PPIs}^{5}$. Additionally, machine learning ${ }^{164,165}$ and data mining ${ }^{166}$ can also be used, facilitating the search of candidate ligand-receptor pairs.

\begin{tabular}{|c|c|c|}
\hline Resource & URL & Ref. \\
\hline \multicolumn{3}{|l|}{ Functional annotations } \\
\hline Gene Ontology & http://geneontology.org/ & 167 \\
\hline UniProt & https://www.uniprot.org/ & 168 \\
\hline KEGG & https://www.genome.jp/kegg/ & 169 \\
\hline \multicolumn{3}{|l|}{ Orthologues } \\
\hline OrthoDB & https://www.orthodb.org/ & 170 \\
\hline gProfiler & https://biit.cs.ut.ee/gprofiler/orth & 171 \\
\hline \multicolumn{3}{|c|}{ Databases of protein-protein interactions } \\
\hline The Human Protein Atlas & https://www.proteinatlas.org/ & 172 \\
\hline STRING & https://string-db.org/ & 173 \\
\hline BioGRID & https://thebiogrid.org/ & 174 \\
\hline PICKLE & http://www.pickle.gr & 175 \\
\hline APID & http://apid.dep.usal.es & 176 \\
\hline IntAct & https://www.ebi.ac.uk/intact & 177 \\
\hline Pathway Commons & http://www.pathwaycommons.org/ & 178 \\
\hline \multicolumn{3}{|l|}{ Ligand-receptor interactions } \\
\hline IUPHAR/BPS Guide to Pharmacology & https://www.guidetopharmacology.org/ & 179 \\
\hline $\begin{array}{l}\text { Resources for cell-cell interactions from } \\
\text { the Bader laboratory }\end{array}$ & http://baderlab.org/CellCelllnteractions & 32 \\
\hline $\begin{array}{l}\text { Compendium of ligand-receptor pairs in } \\
\text { the literature }\end{array}$ & $\begin{array}{l}\text { https://github.com/LewisLabUCSD/ } \\
\text { Ligand-Receptor-Pairs }\end{array}$ & $\begin{array}{l}\text { https://doi.org/10.1038/ } \\
\text { s41576-020-00292-x }\end{array}$ \\
\hline
\end{tabular}

BPS, British Pharmacological Society; IUPHAR, International Union of Basic and Clinical Pharmacology; KEGG, Kyoto Encyclopedia of Genes and Genomes.

points (representing pairs of cells) due to low expression variance of the ligand or the receptor in an interaction is known to bias correlation and can make this score inappropriate ${ }^{95}$.

In addition to these core scoring functions, more advanced approaches can generate interaction scores, such as RNA-Magnet ${ }^{53}$, a method using fuzzy logic to identify candidate ligands and receptors active in cell communication. As mRNAs encoding surface receptors frequently show low abundance, this strategy better accounts for the variance in receptor expression. Other advanced approaches have been wrapped into tools (see the section entitled A growing toolbox to facilitate CCC analysis). Each strategy can decipher CCC but relies on different assumptions that may influence the results. Therefore, knowing the limitations and following best practices in data set preprocessing ${ }^{93,96,97}$ are crucial to minimize false discoveries.

Distinguishing which pair of cells are more likely to interact. Measurement of individual communication scores facilitates the study of CCC, exposing the roles of specific signalling mechanisms; however, it does not reveal the entire interaction state between cells. Thus, it may be desirable to use an aggregate score to define the interactions between pairs of cells ${ }^{84}$.

From the few studies that aggregated communication scores into an overall score (FIC. 2a, step 5), the most common approach quantifies the number of active ligand-receptor pairs between cells (that is, the sum of binary communication scores). This score suggests which cells interact more strongly and enables 
the building of CCC networks to perform graph-based analyses. However, different cell pairs could have similar counts but completely different circuits of communication ${ }^{7}$, causing inaccuracies. Newer methods attempted to deal with those inaccuracies by weighting CCIs and CCC on the basis of additional data. Hence, these approaches enable the comparison of interactions weighted by their importance, among those that are considered active. One approach computes a probability of intracellular communication using a given ligand-receptor pair between a sender and a receiver cell and then aggregates this into a global CCC score ${ }^{25}$. Another study proposed a statistical model to evaluate expression variation of individual genes given a cell's CCIs in its neighbourhood ${ }^{98}$. This method yields coefficients interpretable as an overall CCI score. Here, there is no aggregation of communication scores as the coefficient is computed from the covariance of different factors, such as the distance between cells, the cellular composition of the neighbourhood and transcriptomes including all genes instead of only ligands and receptors. Although these approximations seem more biologically relevant than just counting the expressed ligand-receptor pairs, they require more detailed information that might not be available in all experimental settings.

Alternative strategies to quantify CCC include Euclidian-like or Jaccard-like distance metrics that represent the number of active ligand-receptor pairs in relation to all ligand-receptors in interacting cells. Furthermore, spatial transcriptomics technologies ${ }^{99-104}$ may help train machine learning models to predict a physical distance-dependent potential of CCIs ${ }^{105}$. Expression of ligands and receptors can serve as inputs and distances between cells as outputs.

Although there are many approaches, it remains unclear which metric best captures the underlying biology. Moreover, any strategy relying on gene expression will remain limited to signals captured in the ligand-receptor list, which may miss ways that cells can communicate. Thus, there remains a need for strategies that determine an aggregate CCC potential and functional-spatial relationships of cells.

A growing toolbox to facilitate CCC analysis. In addition to the core scoring functions (FIG. 2b), many tools use advanced statistical methods to identify intercellular communication (TABLE 2). Existing computational tools can be grouped into one of four categories on the basis of the mathematical models used for identifying ligandreceptor interactions, classified here as (1) differential combination-based, (2) network-based, (3) expression permutation-based and (4) array-based tools.

In differential combination-based tools, significantly differentially expressed genes between cell clusters in single-cell RNA-seq data are identified, and these lists are analysed for ligand-receptor pairs. Two such tools, iTALK ${ }^{106}$ and CellTalker ${ }^{61}$, use slightly different downstream analysis methods to curate the final list of significantly interacting ligand-receptor pairs. Similarly, $\mathrm{PyMINEr}^{107}$ annotates interacting cell types but labels interactions as 'activating' or 'inhibitory' according to public interaction databases. Another tool, CCCExplorer $^{67}$, uses differential gene expression and PPI database-guided network analysis of downstream targets and transcription factors to determine activated or deactivated signalling events between two groups of samples or cells. These methods are powerful for discerning ligand-receptor interactions in the background of the rest of the data set but are blind to interactions that are common between all groups.

Network methods are used by several tools and exploit properties of connections between genes. For example, CCCExplorer uses them to identify concerted movements in the expression levels of genes involved in ligand-receptor signalling ${ }^{67}$. The tools SoptSC ${ }^{25}$ and NicheNet ${ }^{54}$ use known interactions between ligands, receptors and downstream targets to build a network of ligand-receptor relationships. The former computes a probability, while the latter uses a personalized PageRank algorithm, in both cases to evaluate the effect of ligand-receptor co-expression on downstream signalling genes in the receiver cell and, thus, obtain a continuous score for ranking ligands and receptors based on this effect. Most recently, a method called 'SpaOTsc'108 formulates intercellular communication as an optimal transport problem ${ }^{109}$ using RNA-seq and spatial information. All these tools use not only the expression levels of ligands and receptors to compute interaction scores but also expression levels of downstream signalling targets, which is intended to be a strength of these techniques. However, they do not account for the rules of gene regulation, so a limitation of these methods is that they are blind to signalling crosstalk, where the signals triggered by one receptor could interfere with the signals triggered by another receptor. Pitfall scenarios could be when an intracellular pathway is highly scored because of the expression of its downstream genes, but its activity may be strongly diminished because of inactivation due to another activated pathway that regulates it with post-translational modifications rather than expression control, as may happen in some cytokine signalling pathways ${ }^{110}$, leading to false positives or false negatives not seen with other strategies.

Expression permutation-based tools are the most widely used among those listed in this Review. These methods compute a communication score for each ligand-receptor pair and evaluate its significance either through cluster label permutation, non-parametric tests to assess differences with the null model, or through empirical methods. To increase confidence, CellPhoneDB $^{30,83}$, CellChat ${ }^{79}$ and ICELLNET ${ }^{84}$ address one of the limitations common to most CCC methods: not considering multisubunit protein complexes. Lists with multimeric proteins are used to assess whether all subunits are simultaneously expressed to identify likely functional ligand-receptor interactions. CellChat also allows multisubunit complexes and incorporates positive and negative effectors into its Hill function-inspired framework. Also, other important features are present in different tools in this category: Giotto ${ }^{111}$ includes spatial expression information, accounting for the potential of cells to interact given their physical proximity, whereas ICELLNET is the only tool that returns a global 
a

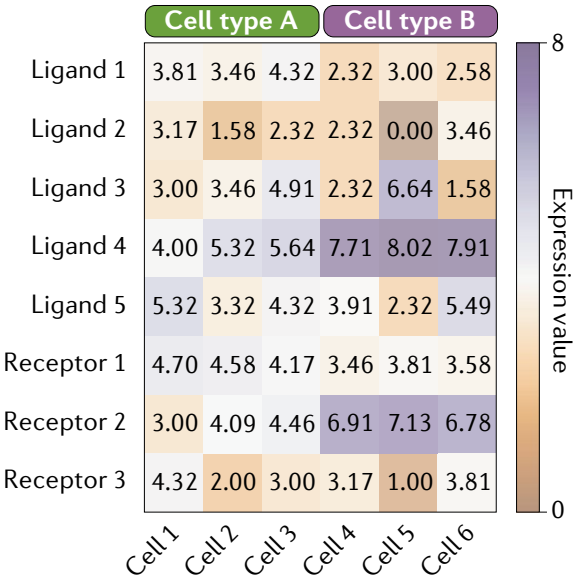

c

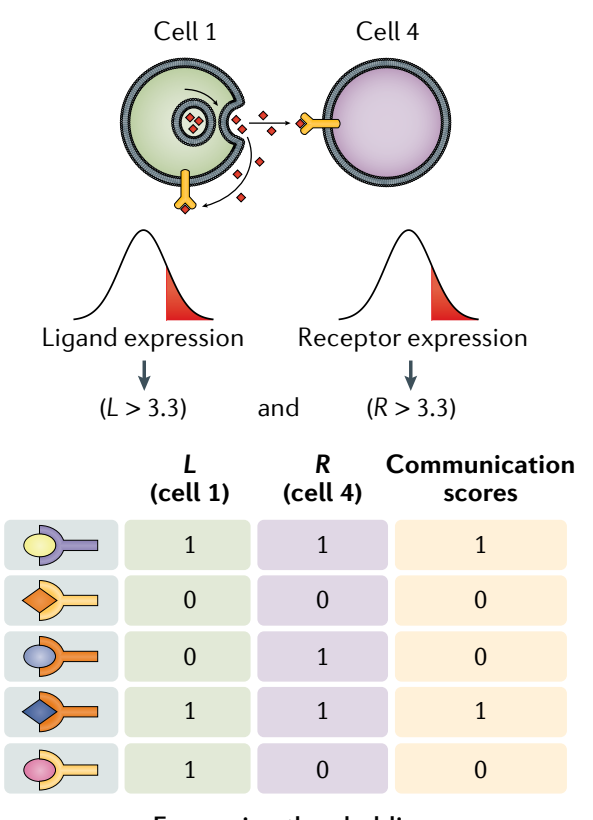

Expression thresholding

e

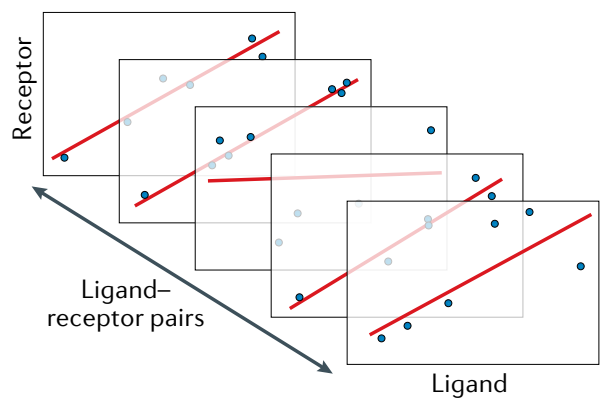

Communication scores

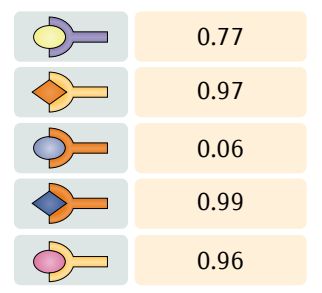

Expression correlation b

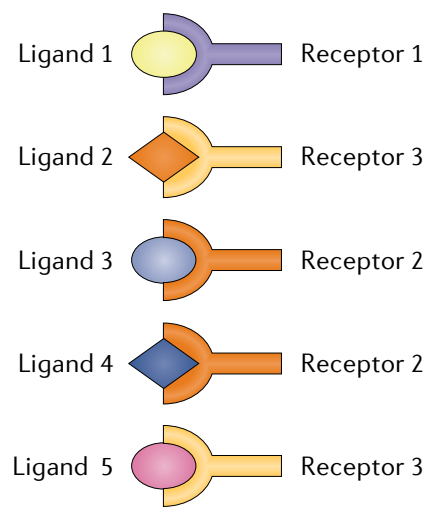

d

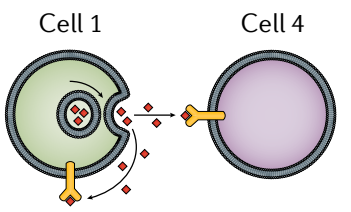

$\bigcap_{\text {Ligand expression }}$

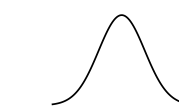

$\stackrel{\downarrow}{\downarrow}$

Receptor expression

$\downarrow$

\begin{tabular}{|c|c|c|c|}
\hline & $\begin{array}{c}\boldsymbol{L} \\
\text { (cell 1) }\end{array}$ & $\begin{array}{c}\boldsymbol{R} \\
\text { (cell 4) }\end{array}$ & $\begin{array}{c}\text { Communication } \\
\text { scores }\end{array}$ \\
\hline \begin{tabular}{|c|c|c|c|}
\hline \\
\hline
\end{tabular} & 3.81 & 3.46 & 13.17 \\
\hline & 3.17 & 3.17 & 10.05 \\
\hline & 3.00 & 6.91 & 20.72 \\
\hline & 4.00 & 6.91 & 27.63 \\
\hline
\end{tabular}

Expression product

f

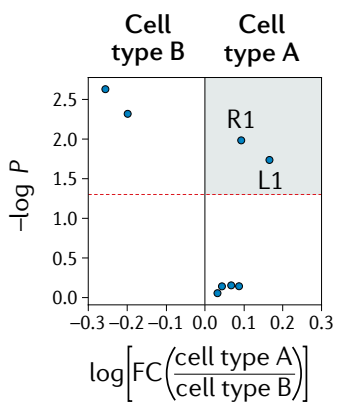

L $\quad$ R Communication (cell A) (cell A) scores

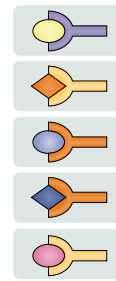

1

1
0
0
0
0

1

0

0

0

0

Differential combinations 
4 Fig. 3 | Toy examples of using core functions to compute communication scores. Two primary inputs are used for quantifying communication scores: a preprocessed gene expression matrix (part a) and a list of interacting proteins to supervise the analysis (for example, ligand-receptor pairs) (part b). Then a communication score (CS) can be computed for every ligand-receptor pair in a given pair of cells. Here, we show how to perform these calculations for four core functions (parts $\mathbf{c}-\mathbf{f}$ ). These are applied to elucidate paracrine (parts c,d) and autocrine (parts e,f) communication. To assess cellcell communication, a CS can be computed for each ligand-receptor pair by accounting for the presence of both partners if their expression is greater than a given threshold, which for demonstrative purposes was set arbitrarily to a value of 3.3 (part c), or by multiplying their expression values (part d). Similarly, the CS for each ligand-receptor pair can be the correlation score obtained from their expression across all cell types for autocrine communication (part e). To reveal non-autocrine interactions, the correlation can be computed across pairs of different cells. Particular signatures of each cell type can be extracted through analysing differentially expressed ligands and receptors. Using the cell type-specific differentially expressed genes, we can assign a binary CS and study the ligand-receptors used for autocrine communication (part f). In this example, autocrine communication is evaluated for cell type $A$ by using its differentially expressed genes with respect to cell type $B$ (cell type A-specific genes are located in the coloured quadrant). Analogously to the correlation score, for non-autocrine communication we would need to consider differentially expressed genes in each of the cell types or samples. For a given pair of cells, we can say that a communication pathway is active when the ligand is differentially expressed in one cell and its cognate receptor is differentially expressed in the other. FC, fold change.

CCC score aggregated from all ligand-receptor interactions using percentile normalization and the expression product core function. SingleCellSignalR ${ }^{112}$ uses a regularized expression product to compute ligand-receptor interaction scores and is the only tool reviewed that provides explicit cut-off values for this score to achieve appropriate false discovery rates based on empirical results.

Tensor-based tools are the most mathematically sophisticated group of tools. Although network computations can be formulated as matrix operations, scTensor ${ }^{113}$ explicitly models ligand-receptor interactions using a tensor. A tensor of rank three is generated from the data, wherein two dimensions are for ligand and receptor expression by each cell type or cluster within the single-cell RNA-seq data set, respectively, and the third dimension represents all ligand-receptor interactions. Then non-negative Tucker decomposition is performed to decompose this tensor ${ }^{14}$, resulting in three matrices with coefficients representing the relationship between interacting cells and their respective ligands and receptors. Thus, this tool captures communication pathways in a context where all pairs of cells are simultaneously considered and extracts the relationships between different CCIs and further produces lower error rates than are obtained with independent hypothesis tests. However, interpreting the scores from a tensor decomposition may not be as straightforward as interpreting the scores from other groups of tools.

The tools described also include powerful visualization features that facilitate the interpretation of results. Several of the more common visualization methods are outlined in FIG. 4 and display data by directly plotting ligand-receptor co-expression patterns and communication scores (FIG. $4 \mathrm{a}-\mathrm{c}$ ) and provide higher-level intuition concerning overall CCI levels and the directionality of these effects between cell types (FIG. $4 d-f$ ). Thus, several tools not only quantify CCIs and CCC but also facilitate their analysis and interpretation.

\section{Assessing predicted CCC mechanisms}

CCC inference should be considered a data-driven process for generating hypotheses, which can lead to different results depending on the strategy adopted (FIG. 3). Thus, validating inferred mechanisms is crucial to confirm associations with phenotypes and behaviours of cell communities. In this section, we discuss current approaches used for this purpose, emphasizing both computational methods to minimize false discoveries and experiments used to validate results, and illustrate hallmark studies that successfully implemented this important step.

Computational minimization of false discoveries. Robust inferences are essential to minimize false positives and false negatives and help reduce the number of validations to perform, which is especially useful when experiments are expensive. From the total pool of inferred ligandreceptor interactions between cells, statistical modelling can assess whether they may result from the null hypothesis and help discard artefactual or non-specific CCC inferences.

Permutation-based analyses can help discard results arising from random noise by prioritizing cell type-enriched ligand-receptor pairs. The cluster labels, representing the cell type, are permuted for each single cell, and the mean gene expression within each permuted cluster is computed, followed by a recalculation of the communication scores. With all communication scores generated for each ligand-receptor pair in a given pair of clusters, a null distribution is built and a $P$ value of the measured communication score can be computed. A full list of tools that use this method is included in the discussion of permutation-based tools (see the section entitled A growing toolbox to facilitate CCC analysis and TABLE 2). As a representative example, the CellPhoneDB tool was applied on single-cell RNA-seq of human first-trimester placentae to understand the regulation of the immune response and how it prevents harmful maternal responses ${ }^{30}$.

Subsampling analysis has been applied to evaluate the robustness of the CCC results. Random subsamples of the original data set are used to rerun the CCC analysis. Using the subsampled results, one can compute true-positive and false-positive rates with respect to the original data set results ${ }^{79}$ and quantify how variable the inferences are, given subtle changes in the composition of cell clusters. CellChat is one tool that has applied this method to compare its performance with that of other tools and measure robustness when identifying the role of a specific population of mouse myeloid cells, termed 'MYL-A', in wound healing through transforming growth factor $-\beta$ signalling ${ }^{79}$.

Enrichment analyses have also helped reduce false discoveries. A recent study applied this strategy to study the role of structural cells (that is, fibroblasts, endothelial cells and epithelial cells) in priming immune responses and how they interact with immune cells ${ }^{58}$. It identified ligand-receptor pairs that were enriched between interacting cell types using a Fisher's exact test over all possible pairs of differentially expressed genes. Supported by other experiments, these interactions revealed the 
Table 2 | Existing tools for measuring cell-cell communication

\begin{tabular}{|c|c|c|c|c|c|c|}
\hline Tool & Method overview & Output & Visualization & Available in & URL & Refs \\
\hline \multicolumn{7}{|c|}{ Differential combinations } \\
\hline CellTalker & $\begin{array}{l}\text { Uses differentially expressed } \\
\text { ligands and receptors in } \\
\text { each cluster to identify } \\
\text { unique interactions } \\
\text { between clusters }\end{array}$ & $\begin{array}{l}\text { Upregulated and } \\
\text { downregulated } \\
\text { interactions between } \\
\text { all clusters }\end{array}$ & $\begin{array}{l}\text { Circos plot of differential } \\
\text { interactions between } \\
\text { clusters }\end{array}$ & $\mathrm{R}$ & $\begin{array}{l}\text { https://github.com/ } \\
\text { arc85/celltalker }\end{array}$ & 61 \\
\hline iTALK & $\begin{array}{l}\text { Enumerates differentially } \\
\text { expressed ligand and } \\
\text { receptor values to identify } \\
\text { LRIs between different } \\
\text { clusters }\end{array}$ & $\begin{array}{l}\text { Upregulated and } \\
\text { downregulated } \\
\text { interactions between } \\
\text { all clusters }\end{array}$ & $\begin{array}{l}\mathrm{CCl} \text { networks, Circos } \\
\text { plots and boxplots }\end{array}$ & $\mathrm{R}$ & $\begin{array}{l}\text { https://github.com/ } \\
\text { Coolgenome/iTALK }\end{array}$ & 106 \\
\hline PyMINEr & $\begin{array}{l}\text { Uses differentially expressed } \\
\text { ligand and receptor pairs to } \\
\text { identify altered signalling } \\
\text { pathways. Detects both } \\
\text { activation and inhibition }\end{array}$ & $\begin{array}{l}\text { Upregulated and } \\
\text { downregulated } \\
\text { interactions between } \\
\text { all clusters }\end{array}$ & $\begin{array}{l}\text { Network visualization } \\
\text { and Circos plots }\end{array}$ & $\begin{array}{l}\text { Python and } \\
\text { standalone } \\
\text { application }\end{array}$ & $\begin{array}{l}\text { https://www. } \\
\text { sciencescott.com/ } \\
\text { pyminer }\end{array}$ & 107 \\
\hline CellPhoneDB & $\begin{array}{l}\text { Randomly permutes cluster } \\
\text { labels to generate a null } \\
\text { distribution of LRI scores } \\
\text { using protein complex } \\
\text { subunit architecture } \\
\text { to identify significant } \\
\text { interactions }\end{array}$ & $\begin{array}{l}\text { Upregulated and } \\
\text { downregulated } \\
\text { interactions between } \\
\text { all clusters }\end{array}$ & $\begin{array}{l}\text { Heatmap of significant } \\
\text { interaction counts, dot } \\
\text { plot of LRIs and cluster } \\
\text { combinations }\end{array}$ & $\begin{array}{l}\text { Python and } \\
\text { Web interface }\end{array}$ & $\begin{array}{l}\text { https://github. } \\
\text { com/Teichlab/ } \\
\text { cellphonedb }\end{array}$ & 30,83 \\
\hline SingleCellSignalR & $\begin{array}{l}\text { Uses a regularized ligand- } \\
\text { receptor expression product } \\
\text { to measure extent of CCC }\end{array}$ & $\begin{array}{l}\text { Interaction scores for } \\
\text { each LRI between all } \\
\text { clusters in the dataset }\end{array}$ & $\begin{array}{l}\text { Circos plot, tables and } \\
\text { graph visualizations of } \\
\text { interactions between } \\
\text { clusters }\end{array}$ & $\mathrm{R}$ & $\begin{array}{l}\text { https://github.com/ } \\
\text { SCA-IRCM }\end{array}$ & 112 \\
\hline \multicolumn{7}{|l|}{ Graph or network } \\
\hline CCCExplorer & $\begin{array}{l}\text { A graph of all signalling } \\
\text { pathways is built, then } \\
\text { a Fisher-like statistic is } \\
\text { computed using ligand, } \\
\text { receptor and downstream } \\
\text { TF expression to identify } \\
\text { significant interactions }\end{array}$ & $\begin{array}{l}\text { Graph visualizations of } \\
\text { all interactions }\end{array}$ & $\begin{array}{l}\text { Interactive directed } \\
\text { graphs }\end{array}$ & $\begin{array}{l}\text { Standalone } \\
\text { application }\end{array}$ & $\begin{array}{l}\text { https://github.com/ } \\
\text { methodistsmab/ } \\
\text { CCCExplorer }\end{array}$ & 67 \\
\hline NicheNet & $\begin{array}{l}\text { A network of ligand- } \\
\text { receptor pathway } \\
\text { interactions is used to } \\
\text { measure the predictive } \\
\text { power of the ligand for } \\
\text { its downstream pathway } \\
\text { targets as an interaction } \\
\text { score, based on a } \\
\text { personalized PageRank } \\
\text { algorithm }\end{array}$ & $\begin{array}{l}\text { Ligand interaction } \\
\text { scores and expressing } \\
\text { cell types for provided } \\
\text { target pathway }\end{array}$ & $\begin{array}{l}\text { Circos plot of } \\
\text { interactions between } \\
\text { cells or clusters }\end{array}$ & $\mathrm{R}$ & $\begin{array}{l}\text { https://github.com/ } \\
\text { saeyslab/nichenetr }\end{array}$ & 54 \\
\hline
\end{tabular}


Table 2 (cont.) | Existing tools for measuring cell-cell communication

\begin{tabular}{|c|c|c|c|c|c|c|}
\hline Tool & Method overview & Output & Visualization & Available in & URL & Refs \\
\hline \multicolumn{7}{|c|}{ Graph or network (cont.) } \\
\hline \multirow[t]{2}{*}{ SoptSC } & \multirow[t]{2}{*}{$\begin{array}{l}\text { Integrates downstream } \\
\text { signalling measurements } \\
\text { into an LRI scoring function }\end{array}$} & \multirow{2}{*}{$\begin{array}{l}\text { Upregulated and } \\
\text { downregulated } \\
\text { interactions between } \\
\text { all clusters }\end{array}$} & \multirow[t]{2}{*}{$\begin{array}{l}\text { Circos plot of } \\
\text { interactions between } \\
\text { cells }\end{array}$} & \multirow[t]{2}{*}{$\begin{array}{l}\text { MATLAB } \\
\text { and R }\end{array}$} & $\begin{array}{l}\text { https://github.com/ } \\
\text { WangShuxiong/ } \\
\text { SoptSC }\end{array}$ & \multirow[t]{2}{*}{25} \\
\hline & & & & & $\begin{array}{l}\text { https://github.com/ } \\
\text { mkarikom/RSoptSC }\end{array}$ & \\
\hline \multicolumn{7}{|c|}{ Tensor based } \\
\hline scTensor & $\begin{array}{l}\text { Tucker decomposition on } \\
\text { a tensor of order three to } \\
\text { identify key LRIs present in } \\
\text { certain cell types }\end{array}$ & $\begin{array}{l}\text { HTML file with } \\
\text { summaries } \\
\text { of clustering, } \\
\text { decomposition } \\
\text { and interaction } \\
\text { components }\end{array}$ & $\begin{array}{l}\text { Many options for } \\
\text { interaction, expression } \\
\text { and pattern visualization }\end{array}$ & $\mathrm{R}$ & $\begin{array}{l}\text { https://github.com/ } \\
\text { rikenbit/scTensor }\end{array}$ & 113 \\
\hline
\end{tabular}

crucial role of structural cells in priming immune response in an organ-specific fashion ${ }^{58}$.

Data generated with other technologies, such as proteomics, have shaped CCC methods and their potential performance. Specifically, they have helped tune the parameters of methods before making predictions on the gene expression data set of interest, addressing issues originating from imperfect mRNA-protein correlations. For example, Browaeys et al. ${ }^{54}$ used several gene and protein expression data sets to optimize NicheNet. Similarly, another study ${ }^{112}$ used gene and protein expression data sets to evaluate false-positive rates and tune the parameters of CCC measurement methods. Thus, integrating other types of data is also important to benchmark and optimize tools.

As discussed here, computational strategies can help reduce false discoveries and facilitate the selection of important results for further research or experimental validation. Although we highlighted cases for which these methods helped drive new hypotheses, many other strategies can be used, such as Wilcoxon's test ${ }^{60}$, random generation of data sets ${ }^{68}$, cross-validation ${ }^{105}$ and Welch's test ${ }^{48}$.

Experimental validation. Many studies use experimental methods to validate CCC mechanisms inferred computationally (TABLE 1; Supplementary Table 1). These cover three levels of experimental tests: (1) confirmation of the expression of proteins thought to be involved in CCC (for example, through proteomics, enzyme-linked immunosorbent assay, western blot or immunohistochemistry); (2) visualization of interactions between gene products expressed in neighbouring cells (for example, through microscopy coupled with immunostaining, single-molecule fluorescence in situ hybridization or measurement of co-occurrence through flow cytometry); and (3) assessment of the functional role of CCC mediators by performing in vivo or in vitro experiments using activators or inhibitors of the interacting molecules or genetic manipulation of cells to observe effects consistent with the predicted communication.
Although providing only tangential information, rather than validation, studies can confirm the presence of proteins to test the potential of signals contributing to CCC events. Immunohistochemistry or western blots have measured the cell type-specific presence of involved signalling complex members. A recent analysis of human liver development used immunohistochemistry to verify VCAM1 production in macrophages and ITGA4 production in erythroid cells of the human fetal liver to suggest a possible interaction between these two cell types and their relevance to lineage differentiation ${ }^{23}$. However, these approaches only support and do not validate a predicted interaction.

Immunohistochemistry and other tagging techniques, such as single-molecule fluorescence in situ hybridization, are frequently used to gain further information about cellular localization for predicted CCIs. This set-up verifies the spatial colocalization of CCC mediators and therefore supports the potential occurrence of a CCC event. For example, in addition to immunostaining to confirm protein expression, statistically significant co-occurrence of IL-33 and its receptor was validated on adjacent AT- 2 cells and basophils, respectively ${ }^{41}$, whereas another study used imaging flow cytometry to show interaction between macrophages and liver progenitor cells ${ }^{23}$. In addition, immunofluorescence was used to colocalize the expression of four ligand-receptor interactions (APOE-LRP1, APOE-LDLR, VTN-KDR and LAMA4-ITGB1) to adjacent cells in mouse brain on embryonic day 14.5 (REF. ${ }^{22}$ ), supporting the relevance of these interactions to the developing brain. Thus, these approaches confirm spatial colocalization of predicted interactions.

Functional assessment is the most informative validation technique. This approach evaluates whether the phenotypes observed in the interacting cells are the result of specific CCC events. For example, the interaction between VEGFA and KDR (also known as VEGFR2) in liver bud development was confirmed by 


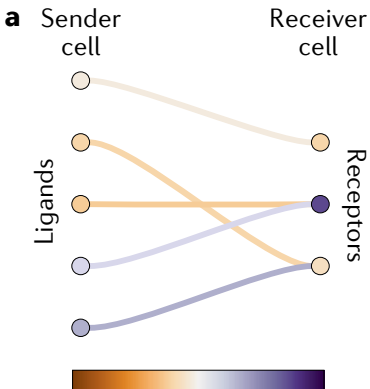

Expression value b

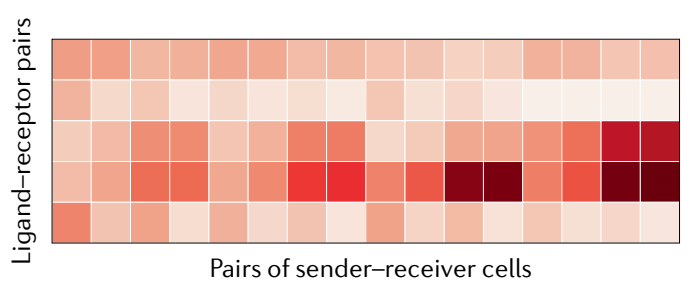

Communication score

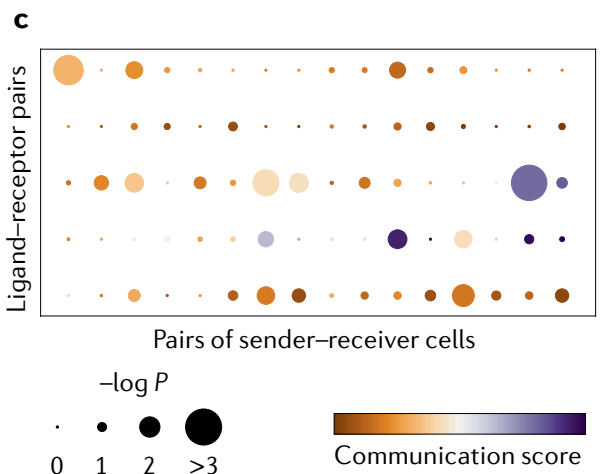

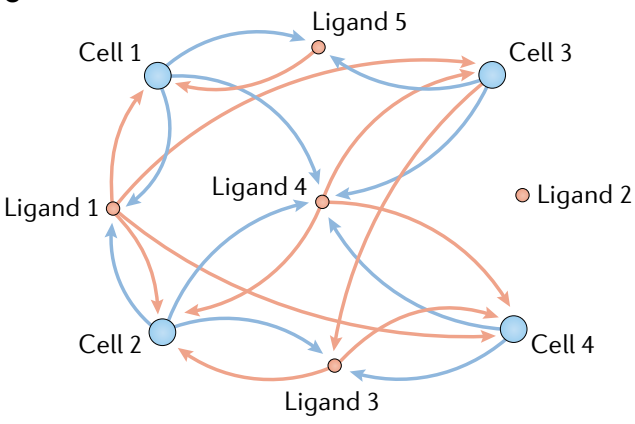

f

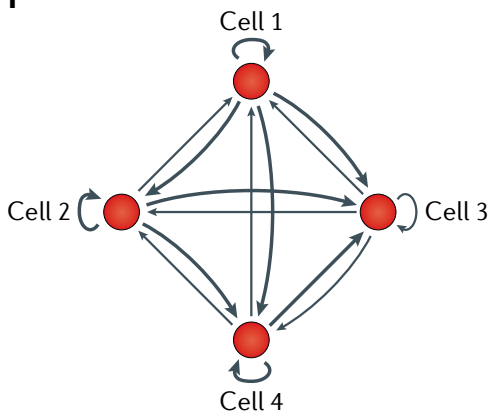

Fig. 4 | Common visualization techniques for cell-cell interactions and communication. a |A Sankey diagram for connecting key ligands from a sender cell to cognate receptors in the receiver cell. Node colour (ligand or receptor) indicates the expression level. b| Heatmap to represent the communication scores for each ligand-receptor interaction in each cell pair. c | Dot plot to show the communication score (colour of dots) and at the same time its significance (size), often obtained from a statistical model or permutation analysis. $\mathbf{d}$ |Circos plot or chord diagram to show key communication pathways used by different cell types to communicate. The links start from a ligand (red) and end in a receptor (blue), which are grouped for each cell type (coloured outer arcs). e | Bipartite network where nodes can be either cells or ligands. Edges can be directed only from a cell to a ligand it produces or from a ligand to a cell that expresses its cognate receptor. $\mathbf{f} \mid$ Cellcell interaction network to represent the potential of cells to interact. Nodes correspond to cells and edges correspond to their interactions. These are directed from a sender cell to a receiver cell, and their thicknesses are proportional to the respective global cell-cell communication scores (for example, number of active ligand-receptor pairs).

dosing KDR inhibitors in vitro into micro-liver buds, significantly reducing hepatoblast abundance and impairing differentiation ${ }^{24}$. Elsewhere, 33 candidate ligands affecting haematopoietic differentiation were dosed in vitro in haematopoietic stem cells, and 27 significantly affected the differentiation rate and trajectory of these cells ${ }^{15}$. Similarly, organ structural cells thought to contribute to the immune response were exposed to candidate cytokines, which induced expression changes similar to those seen upon lymphocytic choriomeningitis virus infection, as determined with a mouse model of lymphocytic choriomeningitis virus infection as a reference $^{58}$.

\section{Challenges and future directions}

As the methods measuring CCIs improve and the associated results are experimentally validated, new research opportunities will arise that may improve the reliability of inferred CCIs. Furthermore, novel insights will emerge through the study of interactions between cells from different species and the engineering of phenotypes by manipulating communication events.
Multi-omics integration. Although mRNA and protein levels are qualitatively correlated, transcriptomics may not represent a fully accurate view of intercellular communication, as transcript and protein abundances can be uncoupled by post-transcriptional and posttranslational processes ${ }^{82,115,116}$. For example, the inferred presence of ligand-receptor pairs from transcriptomics may not coincide with their actual presence in proteomic data $^{7,82}$. Borrowing information from other omics technologies can improve confidence in results ${ }^{16,117}$, especially for emerging technologies such as Nativeomics ${ }^{118}$, which detects intact ligand-receptor assemblies using mass spectrometry, and INs-seq ${ }^{119}$, which couples singlecell RNA-seq with intracellular protein measurements to simultaneously profile transcription factors, signalling activity and metabolism. Moreover, emerging technologies such as single-cell proteomics ${ }^{11}$ will become important inputs for CCC methods and complement single-cell RNA-seq to improve CCI predictions.

Omics integration can extend beyond gene expression. Mammalian cells are covered by a thick glycocalyx, and most hormones and receptors are glycoproteins. 
Hence, glycomic data can add contextual information to CCI analyses. Glycosylation has an impact on protein interactions, especially in ligand-receptor binding as many ligands are glycosylated ${ }^{120}$, and glycosylation can change receptor affinity ${ }^{121-123}$. This phenomenon may rewire CCIs and affect, for example, development or $\mathrm{T}$ cell activation ${ }^{124}$. Moreover, glycans are involved in interspecies crosstalk, contributing to the specificity of communication ${ }^{125-127}$. Therefore, integration of additional omics technologies, such as proteomics and glycomics, will help identify additional CCIs that are missed with use of RNA-based methods.

Adding a spatial dimension. Ligand mRNA expression and ligand abundance are not the only factors required for communication: the ligand must also be localized in the correct cellular compartment, which RNA-based methods are blind to, and interacting cells are usually close to each other, which routine single-cell RNA-seq experiments cannot measure. To improve reliability of computed CCIs, it is crucial to account for spatial positions of mediators and interacting cells. Single-cell analyses have considered cell location in mouse bone marrow ${ }^{53,128}$, demonstrating that cell proximity is key to the study of intercellular communication. The study of spatial proximity in interacting cells is an emerging approach ${ }^{129}$. One technology for profiling these physical interactions, PIC-seq, uses cell sorting to acquire and transcriptionally profile physically interacting cells (PICs) through massively parallel single-cell RNA-seq ${ }^{130}$. The study authors presented an algorithm for deconvolving the data to capture signals from intercellular physical interactions. One advantage of this method is that it captures more details in the crosstalk of PICs than single-cell RNA-seq. Although this technology is promising, it is currently limited to studying PICs from only two populations of cells. Nevertheless, similarly to other PIC-based methods ${ }^{128,131,132}$, PIC-seq can be readily integrated into the pipelines described here and leverage the analysis of CCIs by including the intrinsic spatial information that PICs encode. As more approaches emerge for spatial-based transcriptomics ${ }^{99-104}$ and proteomics ${ }^{133,134}$, future studies and algorithms should include this information. Accounting for the physical distance between cells will lead to the generation of new scoring functions that may better capture the potential of cells to communicate and interact ${ }^{53,98,108,111,135}$. As an example, ligand-specific diffusion constants can be considered to reflect how effectively gene products can mediate long-distance communication ${ }^{136,137}$.

Adding a temporal dimension. Time is another important factor for studying CCC. It can help elucidate how communication evolves and detect important changes in dynamic processes, such as cellular differentiation and the immune response. One can use samples taken at different time points or infer pseudotimes from the RNA-seq data set as happens when one is studying cellular differentiation through lineage tracing ${ }^{138,139}$. Then CCC analyses on each (pseudo) time point can identify active ligandreceptor pairs and test the overall interaction potential between cells ${ }^{25}$. However, time-dependent behaviours might be uncoupled from mRNA levels, hindering the detection of changes through transcriptomics-based CCC predictions. mRNA expression can be temporarily disconnected from the activity of the products owing to long half-lives or storage of the products ${ }^{140}$. It can also take longer to reach the appropriate abundance of a ligand or a receptor than the production of the cognate $\mathrm{mRNA}^{82}$. For example, endothelial cells store cell adhesion molecules, such as P-selectins, in granules, which are quickly mobilized to the cell surface to start the recruitment of immune cells, instead of expressing those proteins de novo ${ }^{141}$. Thus, time-dependent analyses can improve CCC discoveries, but other data types should be integrated to distinguish whether phenotypes stem from CCC or other dynamics of gene expression.

\section{Shedding light on interspecies interactions.} Comprehensive lists of ligand-receptor pairs or interacting surface-secreted proteins are crucial for algorithms that study intraspecies CCIs. However, study of interspecies interactions - for example, humans and pathogens ${ }^{142-145}$ - requires lists of molecular interactions between species. An opportunity awaits to define interspecies PPI lists with omics methods. Considering the avalanche of data generated in the microbiome field $^{146-149}$ and recent approaches for modelling microbial communities ${ }^{150}$, lists of interacting proteins or crossspecies ligand-receptor pairs to enable CCI analyses would evidently yield novel discoveries. For example, a study mapped the interaction between inclusion membrane proteins secreted by Chlamydia trachomatis and cognate human proteins ${ }^{145}$, providing insights into the host machinery this pathogen uses to establish the intracellular niche needed for infection. Thus, even smallscale lists of interacting partners between the host and a single microorganism, instead of the whole microbiota, will open great opportunities to use CCC methods to understand infections and diseases on the basis of host-pathogen interactions ${ }^{151,152}$. Although this endeavour will require validation of putative interactions, as done for human-virus protein interactions ${ }^{153-156}$, results from such studies will have a considerable impact on biomedicine and microbiome fields.

Predicting and manipulating phenotypes. Models for predicting phenotypes were previously trained with the active communication pathways underlying intercellular interactions ${ }^{71}$. As more models are developed and their predictive power is increased, they will enable the identification of drug targets and manipulation of phenotypes through cell engineering. Particularly, removing or adding communication pathways with genome editing and cell engineering technologies ${ }^{157}$ will modify cellular phenotypes to control how pairs of cells interact. This approach will have a great impact on many fields, such as developmental biology ${ }^{158}$, wherein CCIs are fundamental for cell differentiation into specific lineages. Biomedicine and biotherapeutics will benefit further from controlling CCIs, particularly in modifying disease courses, as is currently done with immune checkpoint inhibitors ${ }^{159,160}$. As a proof of concept, a tool that induces gene activation when specific cells interact or are directly in contact was recently built by combining a synthetic Notch receptor 
and the CRISPR-Cas9 system ${ }^{161}$. This biological device enabled customization of cell behaviours as observed through the expression of reporter genes. As reporters may be replaced by activators or inhibitors of other communication pathways, this tool holds great potential for therapeutic uses. Thus, manipulation of interaction pathways to control CCIs is feasible, with predictive models helping to decide which modifications to perform.

\section{Conclusions}

Incredible advances are now emerging to infer CCIs and CCC from gene expression. The diverse strategies applied have elucidated fundamental roles of cells within their communities and how they shape cellular functions, with great potential for future applications, especially in biomedicine and biotherapeutics. Each approach for inferring CCIs and CCC has its own assumptions and limitations to consider; when one is using such strategies, it is important to be aware of these strengths and weaknesses and to choose appropriate parameters for analyses. Methodological and technological challenges remain, but many opportunities exist to increase our understanding of intercellular interactions.

Published online 9 November 2020
1. Zhou, X. et al. Circuit design features of a stable two-cell system. Cell 172, 744-757.e17 (2018). Rouault, H. \& Hakim, V. Different cell fates from cell-cell interactions: core architectures of two-cell bistable networks. Biophys. J. 102, 417-426 (2012).

3. Bonnans, C., Chou, J. \& Werb, Z. Remodelling the extracellular matrix in development and disease. Nat. Rev. Mol. Cell Biol. 15, 786-801 (2014).

4. Bich, L., Pradeu, T. \& Moreau, J.-F. Understanding multicellularity: the functional organization of the intercellular space. Front. Physiol. 10, 1170 (2019).

5. Rao, V. S., Srinivasa Rao, V., Srinivas, K., Sujini, G. N. ¿ Sunand Kumar, G. N. Protein-protein interaction detection: methods and analysis. Int. J. Proteom. 2014, 1-12 (2014).

6. Zhou, Y. et al. Evaluation of single-cell cytokine secretion and cell-cell interactions with a hierarchical loading microwell chip. Cell Rep. 31, 107574 (2020). Ramilowski, J. A. et al. A draft network of ligandreceptor-mediated multicellular signalling in human. Nat. Commun. 6, 7866 (2015).

This study provides a list of human ligand-receptor pairs that has been broadly used as a reference. It also evaluates the false-positive rate of using different thresholds for computing communication scores by expression thresholding.

8. Mortazavi, A., Williams, B. A., McCue, K., Schaeffer, L. $\&$ Wold, B. Mapping and quantifying mammalian transcriptomes by RNA-Seq. Nat. Methods 5 621-628 (2008)

9. Fend, F et al. Immuno-LCM: laser capture microdissection of immunostained frozen sections for mRNA analysis. Am. J. Pathol. 154, 61-66 (1999).

10. Tang, F. et al. mRNA-Seq whole-transcriptome analysis of a single cell. Nat. Methods 6, 377-382 (2009).

11. Marx, V. A dream of single-cell proteomics. Nat. Methods 16, 809-812 (2019).

12. Grün, D. et al. Single-cell messenger RNA sequencing reveals rare intestinal cell types. Nature 525, 251-255 (2015)

13. Park, J.-E. et al. A cell atlas of human thymic development defines $\mathrm{T}$ cell repertoire formation. Science 367, eaay3224 (2020)

14. Kirouac, D. C. et al. Dynamic interaction networks in a hierarchically organized tissue. Mol. Syst. Biol. 6, 417 (2010).

15. Qiao, W. et al. Intercellular network structure and regulatory motifs in the human hematopoietic system. Mol. Syst. Biol. 10, 741 (2014).

This work reports the construction of a CCC network that enables the discovery of cellula properties associated with the production of ligands and receptors.

16. Yuzwa, S. A. et al. Proneurogenic ligands defined by modeling developing cortex growth factor communication networks. Neuron 91, 988-1004 (2016).

17. Paik, D. T. et al. Large-scale single-cell RNA-seq reveals molecular signatures of heterogeneous populations of human induced pluripotent stem cell-derived endothelial cells. Circ. Res. 123 443-450 (2018)

18. Li, G. et al. Single cell expression analysis reveals anatomical and cell cycle-dependent transcriptional shifts during heart development. Development 146 dev173476 (2019).

19. Schiebinger, G. et al. Optimal-transport analysis of single-cell gene expression identifies developmental trajectories in reprogramming. Cell 176, 928-943.e22 (2019).
20. Xue, Y. et al. A 3D atlas of hematopoietic stem and progenitor cell expansion by multi-dimensional RNA-sea analysis. Cell Rep. 27, 1567-1578.e5 (2019).

21. Basson, M. A. Signaling in cell differentiation and morphogenesis. Cold Spring Harb. Perspect. Biol. 4 a008151 (2012)

22. Sheikh, B. N. et al. Systematic identification of cell-cell communication networks in the developing brain iScience 21, 273-287 (2019).

23. Popescu, D.-M et al. Decoding human fetal liver haematopoiesis. Nature 574, 365-371 (2019).

24. Camp, J. G. et al. Multilineage communication regulates human liver bud development from pluripotency. Nature 546, 533-538 (2017). This study reports the analysis of intercellular communication from gene expression during the development of an organoid.

25. Wang, S., Karikomi, M., MacLean, A. L. \& Nie, Q. Cell lineage and communication network inference via optimization for single-cell transcriptomics. Nucleic Acids Res. 47, e66 (2019).

26. Joost, S. et al. Single-cell transcriptomics reveals that differentiation and spatial signatures shape epiderma and hair follicle heterogeneity. Cell Syst. 3, 221-237.e9 (2016).

27. Joost, S. et al. Single-cell transcriptomics of traced epidermal and hair follicle stem cells reveals rapid adaptations during wound healing. Cell Rep. 25, 585-597.e7 (2018).

28. Wang, S. et al. Single cell transcriptomics of human epidermis reveals basal stem cell transition states. Preprint at bioRxiv https://doi.org/10.1101/784579 (2019).

29. Pavlic ev, M. et al. Single-cell transcriptomics of the human placenta: inferring the cell communication network of the maternal-fetal interface. Genome Res. 27, 349-361 (2017).

First report using gene expression for inferring CCC in the placenta.

30. Vento-Tormo, R. et al. Single-cell reconstruction of the early maternal-fetal interface in humans. Nature 563 347-353 (2018)

This study reports the development of CellPhoneDB, one of the most used tools for deciphering CCC and the first to consider multimeric proteins. This strategy for analysing ligand-receptor interactions was applied to the maternal-fetal interface.

31. Arneson, D. et al. Single cell molecular alterations reveal target cells and pathways of concussive brain injury. Nat. Commun. 9, 3894 (2018).

32. Ximerakis, M. et al. Single-cell transcriptomic profiling of the aging mouse brain. Nat. Neurosci. 22 1696-1708 (2019).

33. Skelly, D. A. et al. Single-cell transcriptional profiling reveals cellular diversity and intercommunication in the mouse heart. Cell Rep. 22, 600-610 (2018).

34. Wang, L. et al. Single-cell reconstruction of the adult human heart during heart failure and recovery reveals the cellular landscape underlying cardiac function Nat Cell Biol. 22, 108-119 (2020).

35. Wu, H. et al. Single-cell transcriptomics of a human kidney allograft biopsy specimen defines a diverse inflammatory response. J. Am. Soc. Nephrol. 29, 2069-2080 (2018).

36. Wu, H., Kirita, Y. Donnelly, E. L. \& Humphreys, B. D. Advantages of single-nucleus over single-cell RNA sequencing of adult kidney: rare cell types and nove cell states revealed in fibrosis. J. Am. Soc. Nephrol. 30, 23-32 (2019).
37. Stewart, B. J. et al. Spatiotemporal immune zonation of the human kidney. Science 365, 1461-1466 (2019).

38. Ding, C. et al. A cell-type-resolved liver proteome Mol. Cell. Proteom. 15, 3190-3202 (2016).

39. Bonnardel, J. et al. Stellate cells, hepatocytes, and endothelial cells imprint the Kupffer cell identity on monocytes colonizing the liver macrophage niche. Immunity 51, 638-654.e9 (2019).

40. Zepp, J. A et al. Distinct mesenchymal lineages and niches promote epithelial self-renewal and myofibrogenesis in the lung. Cell 170, 1134-1148.e10 (2017).

41. Cohen, M. et al. Lung single-cell signaling interaction map reveals basophil role in macrophage imprinting. Cell 175, 1031-1044.e18 (2018).

42. Raredon, M. S. B. et al. Single-cell connectomic analysis of adult mammalian lungs. Sci. Adv. $\mathbf{5}$ eaaw3851 (2019).

43. Niethamer, T. K. et al. Defining the role of pulmonary endothelial cell heterogeneity in the response to acute lung injury. eLife 9, e53072 (2020)

44. Suryawanshi, H., Morozov, P., Straus, A. \& Sahasrabudhe, N. A single-cell survey of the human first-trimester placenta and decidua. Sci. Adv. https://doi.org/10.1126/sciadv.aau4788 (2018).

45. Hu, Y. et al. Dissecting the transcriptome landscape of the human fetal neural retina and retinal pigment epithelium by single-cell RNA-seq analysis. PLOS Biol. 17, e3000365 (2019).

46. Hrvatin, S. et al. Single-cell analysis of experiencedependent transcriptomic states in the mouse visual cortex. Nat. Neurosci. 21, 120-129 (2018).

47. Solé-Boldo, L. et al. Single-cell transcriptomes of the human skin reveal age-related loss of fibroblast priming. Commun. Biol. 3, 188 (2020).

48. Fernandez, D. M. et al. Single-cell immune landscape of human atherosclerotic plaques. Nat. Med. 25, 1576-1588 (2019).

49. Martin, J. C. et al. Single-cell analysis of Crohn's disease lesions identifies a pathogenic cellular module associated with resistance to anti-TNF therapy. Cell 178, 1493-1508.e20 (2019)

50. Xiong, X. et al. Landscape of intercellular crosstalk in healthy and NASH liver revealed by single-cell secretome gene analysis. Mol. Cell 75, 644-660.e5 (2019).

51. Vieira Braga, F. A. et al. A cellular census of human lungs identifies novel cell states in health and in asthma. Nat. Med. 25, 1153-1163 (2019).

52. Qi, F., Qian, S., Zhang, S. \& Zhang, Z. Single cell RNA sequencing of 13 human tissues identify cell types and receptors of human coronaviruses. Biochem. Biophys. Res. Commun. https://doi.org/10.1016/j.bbrc.2020. 03.044 (2020).

53. Baccin $C$ et al Combined single-cell and spatial transcriptomics reveal the molecular, cellular and spatial bone marrow niche organization. Nat. Cell Biol. 22, 38-48 (2020).

54. Browaeys, R., Saelens, W. \& Saeys, Y. NicheNet: modeling intercellular communication by linking ligands to target genes. Nat. Methods https://doi.org/ 10.1038/s41592-019-0667-5 (2019). This study introduces a PageRank-based algorithm to rank ligand-receptor interactions involved in communication of cells.

55. Cain, M. P., Hernandez, B. J. \& Chen, J. Quantitative single-cell interactomes in normal and virus-infected mouse lungs. Preprint at bioRxiv https://doi.org/ 10.1101/2020.02.05.936054 (2020). 
56. Chua, R. L. et al. COVID-19 severity correlates with airway epithelium-immune cell interactions identified by single-cell analysis. Nat. Biotechnol. https://doi.org 10.1038/s41587-020-0602-4 (2020).

57. Rieckmann, J. C. et al. Social network architecture of human immune cells unveiled by quantitative proteomics. Nat Immunol 18,583-593 (2017).

58. Krausgruber, T. et al. Structural cells are key regulators of organ-specific immune responses. Nature https://doi.org/10.1038/s41586-020-2424-4 (2020).

59. Puram, S. V. et al. Single-cell transcriptomic analysis of primary and metastatic tumor ecosystems in head and neck cancer. Cell 171, 1611-1624.e24 (2017).

60. Kumar, M. P. et al. Analysis of single-cell RNA-seq identifies cell-cell communication associated with tumor characteristics. Cell Rep. 25, 1458-1468.e4 (2018).

This study is an example of using expression products for measuring intercellular communication and for finding relationships between ligandreceptor pairs and tumour phenotypes.

61. Cillo, A. R. et al. Immune landscape of viral- and carcinogen-driven head and neck cancer. Immunity https://doi.org/10.1016/j.immuni.2019.11.014 (2019).

62. Zhang, Q. et al. Landscape and dynamics of single immune cells in hepatocellular carcinoma. Cell $\mathbf{1 7 9}$ 829-845.e20 (2019)

63. Song, Q. et al. Dissecting intratumoral myeloid cell plasticity by single cell RNA-seq. Cancer Med. 68, 7 (2019).

64. Zhang, M. et al. Single cell transcriptomic architecture and intercellular crosstalk of human intrahepatic cholangiocarcinoma. J. Hepatol. https://doi.org/ 10.1016/j.jhep.2020.05.039 (2020)

65. Finotello, F., Rieder, D., Hackl, H. \& Trajanoski, Z. Next-generation computational tools for interrogating cancer immunity. Nat. Rev. Genet. 20, 724-746 (2019).

66. Zitvogel, L. \& Kroemer, G. Targeting PD-1/PD-L1 interactions for cancer immunotherapy. Oncoimmunology 1, 1223-1225 (2012).

67. Choi, H. et al. Transcriptome analysis of individual stromal cell populations identifies stroma-tumor crosstalk in mouse lung cancer model. Cell Rep. 10, 1187-1201 (2015)

68. Yeung, T.-L. et al. Systematic identification of druggable epithelial-stromal crosstalk signaling networks in ovarian cancer. J. Natl. Cancer Inst. 111 272-282 (2019)

69. Graeber, T. G. \& Eisenberg, D. Bioinformatic identification of potential autocrine signaling loops in cancers from gene expression profiles. Nat. Genet. 29 295-300 (2001)

This study is an early example of inferring communication of cancer cells from gene expression of ligand-receptor pairs. It also introduces the use of correlation as a communication score.

70. Zhou, J. X., Taramelli, R., Pedrini, E., Knijnenburg, T. \& Huang, S. Extracting intercellular signaling network of cancer tissues using ligand-receptor expression patterns from whole-tumor and single-cell transcriptomes. Sci. Rep. 7, 8815 (2017)

71. Yuan, D., Tao, Y., Chen, G. $\&$ Shi, T. Systematic expression analysis of ligand-receptor pairs reveals important cell-to-cell interactions inside glioma. Cell Commun. Signal. 17, 48 (2019). This is pioneering work that trains a machine learning model to predict the prognosis of patients with glioma from the ligand-receptor pairs used by glioma cells.

72. Murali, T. et al. DrolD 2011: a comprehensive, integrated resource for protein, transcription factor, RNA and gene interactions for Drosophila. Nucleic Acids Res. 39, D736-D743 (2011).

73. Thurmond, J. et al. FlyBase 2.0: the next generation. Nucleic Acids Res. 47, D759-D765 (2019).

74. Girard, L. R. et al. WormBook: the online review of Caenorhabditis elegans biology. Nucleic Acids Res. 35, D472-D475 (2007)

75. Harris, T. W. et al. WormBase: a comprehensive resource for nematode research. Nucleic Acids Res. 38, D463-D467 (2010)

76. Oh, E.-Y. et al. Extensive rewiring of epithelial-stromal co-expression networks in breast cancer. Genome Biol. 16, 128 (2015)

77. Han, X. et al. Mapping the mouse cell atlas by microwell-seq. Cell 172, 1091-1107.e17 (2018).

78. Krämer, A., Green, J., Pollard, J. Jr \& Tugendreich, S Causal analysis approaches in ingenuity pathway analysis. Bioinformatics 30, 523-530 (2014).
79. Jin, S. et al. Inference and analysis of cell-cell communication using CellChat. Preprint at bioRxiv https://doi.org/10.1101/2020.07.21.214387 (2020).

80. Wrana, J. L. et al. TGF $\beta$ signals through a heteromeric protein kinase receptor complex. Cell 71, 1003-1014 (1992).

81. Sato, N. \& Miyajima, A. Multimeric cytokine receptors common versus specific functions. Curr. Opin. Cell Biol. 6, 174-179 (1994).

82. Buccitelli, C. \& Selbach, M. mRNAs, proteins and the emerging principles of gene expression control. Nat. Rev. Genet. https://doi.org/10.1038/s41576020-0258-4 (2020).

83. Efremova, M., Vento-Tormo, M., Teichmann, S. A. $\S$ Vento-Tormo, R. CellPhoneDB: inferring cell-cell communication from combined expression of multi-subunit ligand-receptor complexes. Nat. Protoc https://doi.org/10.1038/s41596-020-0292-x (2020).

84. Noël, F. et al. ICELLNET: a transcriptome-based framework to dissect intercellular communication. Preprint at bioRxiv https://doi.org/10.1101/2020.03. 05.976878 (2020)

85. Komurov, K. Modeling community-wide molecular networks of multicellular systems. Bioinformatics $\mathbf{2 8}$, 694-700 (2012)

86. Richelle, A., Joshi, C. \& Lewis, N. E. Assessing key decisions for transcriptomic data integration in biochemical networks. PLoS Comput. Biol. 15 e1007185 (2019).

87. Richelle, A. et al. What does your cell really do? Model-based assessment of mammalian cells metabolic functionalities using omics data. Preprint at bioRxiv https://doi.org/10.1101/2020.04.26.057943 (2020).

88. Costa-Silva, J., Domingues, D. \& Lopes, F. M. RNA-Seq differential expression analysis: an extended review and a software tool. PLOS ONE 12, e0190152 (2017)

89. Wang, T., Li, B., Nelson, C. E. \& Nabavi, S. Comparative analysis of differential gene expression analysis tools for single-cell RNA sequencing data. BMC Bioinformatics 20, 40 (2019).

90. Maedler, K et al. Low concentration of interleukin1 beta induces FLICE-inhibitory protein-mediated beta-cell proliferation in human pancreatic islets. Diabetes 55, 2713-2722 (2006).

91. Middendorf, T. R. \& Aldrich, R. W. The structure of binding curves and practical identifiability of equilibrium ligand-binding parameters. J. Gen. Physiol. 149, 121-147 (2017)

92. Goldman, S. L. et al. The impact of heterogeneity on single-cell sequencing. Front. Genet. 10, 8 (2019).

93. AlJanahi, A. A., Danielsen, M. \& Dunbar, C. E. An introduction to the analysis of single-cell RNAsequencing data. Mol. Ther. Methods Clin. Dev. 10, 189-196 (2018)

94. Lähnemann, D. et al. Eleven grand challenges in single-cell data science. Genome Biol 21, 31 (2020).

95. Aggarwal, R. \& Ranganathan, P. Common pitfalls in statistical analysis: the use of correlation techniques. Perspect. Clin. Res. 7, 187-190 (2016).

96. Conesa, A. et al. A survey of best practices for RNA-seq data analysis. Genome Biol. 17, 13 (2016).

97. Luecken, M. D. \& Theis, F. J. Current best practices in single-cell RNA-seq analysis: a tutorial. Mol. Syst. Biol. 15, e8746 (2019).

98. Arnol, D., Schapiro, D., Bodenmiller, B., SaezRodriguez, J. \& Stegle, O. Modeling cell-cell interactions from spatial molecular data with spatial variance component analysis. Cell Rep. 29, 202-211.e6 (2019).

In this pioneering work, the authors evaluated gene expression variability due to CCls by integrating spatial transcriptomics.

99. Satija, R., Farrell, J. A., Gennert, D., Schier, A. F. \& Regev, A. Spatial reconstruction of single-cell gene expression data. Nat. Biotechnol. 33, 495-502 (2015).

100. Navarro, J. F., Sjöstrand, J., Salmén, F., Lundeberg, J. ¿ Ståhl, P. L. ST Pipeline: an automated pipeline for spatial mapping of unique transcripts. Bioinformatics 33, 2591-2593 (2017)

101. Rodriques, S. G. et al. Slide-seq: a scalable technology for measuring genome-wide expression at high spatial resolution. Science 363, 1463-1467 (2019).

102. Vickovic, S. et al. High-definition spatial transcriptomics for in situ tissue profiling. Nat. Methods 16, 987-990 (2019).

103. Moncada, R. et al. Integrating microarray-based spatial transcriptomics and single-cell RNA-seq reveals tissue architecture in pancreatic ductal adenocarcinomas. Nat. Biotechnol. https://doi.org/ 10.1038/s41587-019-0392-8 (2020).
104. Ståhl, P. L. et al. Visualization and analysis of gene expression in tissue sections by spatial transcriptomics. Science 353, 78-82 (2016).

105. Li, D., Ding, J. \& Bar-Joseph, Z. Identifying signaling genes in spatial single cell expression data. Preprint at bioRxiv https://doi.org/10.1101/2020.07.27.221465 (2020).

106. Wang, Y. et al. iTALK: an R package to characterize and illustrate intercellular communication. Preprint at bioRxiv https://doi.org/10.1101/507871 (2019).

107. Tyler, S. R. et al. PyMINEr finds gene and autocrineparacrine networks from human islet scRNA-Seq. Cell Rep. 26, 1951-1964.e8 (2019).

108. Cang, Z. \& Nie, Q. Inferring spatial and signaling relationships between cells from single cell transcriptomic data. Nat. Commun. 11, 2084 (2020).

109. Titouan, V., Courty, N., Tavenard, R., Laetitia, C. \& Flamary, R. in Proceedings of the 36th International Conference on Machine Learning vol. 97 (eds Chaudhuri, K. \& Salakhutdinov, R.) 6275-6284 (PMLR, 2019).

110. Yasukawa, H. Sasaki, A. \& Yoshimura, A. Negative regulation of cytokine signaling pathways. Annu. Rev. Immunol. 18, 143-164 (2000).

111. Dries, R. et al. Giotto, a pipeline for integrative analysis and visualization of single-cell spatial transcriptomic data. Preprint at bioRxiv https://doi. org/10.1101/701680 (2019).

112. Cabello-Aguilar, S. et al. SingleCellSignalR: inference of intercellular networks from single-cell transcriptomics. Nucleic Acids Res. 48, e55 (2020)

113. Tsuyuzaki, K., Ishii, M. \& Nikaido, I. Uncovering hypergraphs of cell-cell interaction from single cell RNA-sequencing data. Preprint at bioRxiv https://doi. org/10.1101/566182 (2019).

This is the first work using tensor decomposition for inferring CCls from gene expression.

114. Kim, Y. \& Choi, S. in 2007 IEEE Conference on Computer Vision and Pattern Recognition 1-8 (IEEE, 2007).

115. Liu, Y., Beyer, A. \& Aebersold, R. On the dependency of cellular protein levels on mRNA abundance. Cell 165, 535-550 (2016).

116. Wegler, C. et al. Global variability analysis of mRNA and protein concentrations across and within human tissues. NAR Genom. Bioinform. 2, lqz010 (2019).

117. Grandclaudon, M. et al. A quantitative multivariate model of human dendritic cell-T helper cell communication. Cell 179, 432-447.e21 (2019).

118. Gault, J. et al. Combining native and 'omics' mass spectrometry to identify endogenous ligands bound to membrane proteins. Nat. Methods 17, 505-508 (2020).

119. Katzenelenbogen, Y. et al. Coupled scRNA-seq and intracellular protein activity reveal an immunosuppressive role of TREM2 in cancer. Cell https://doi.org/10.1016/j.cell.2020.06.032 (2020).

120. Madsen, T. D. et al. An atlas of O-linked glycosylation on peptide hormones reveals diverse biological roles. Nat. Commun. 11, 4033 (2020).

121. Arey, B. J. in Glycosylation (ed. Petrescu, S.) (InTech, 2012)

122. Lux, A. \& Nimmerjahn, F. in Crossroads between Innate and Adaptive Immunity III 113-124 (Springer, 2011).

123. Boscher, C., Dennis, J. W. \& Nabi, I. R. Glycosylation, galectins and cellular signaling. Curr. Opin. Cell Biol. 23, 383-392 (2011)

124. De Bousser, E., Meuris, L., Callewaert, N. \& Festjens, N. Human $T$ cell glycosylation and implications on immune therapy for cancer. Hum. Vaccin. Immunother. https://doi.org/10.1080/21645515.2020.1730658 (2020).

125. Beltrao, P., Bork, P., Krogan, N. J. \& van Noort, V. Evolution and functional cross-talk of protein posttranslational modifications. Mol. Syst. Biol. 9, 714 (2013).

126. Tytgat, H. L. P. \& de Vos, W. M. Sugar coating the envelope: glycoconjugates for microbe-host crosstalk. Trends Microbiol. 24, 853-861 (2016).

127. Gagneux, P., Aebi, M. \& Varki, A. in Essentials of Glycobiology (eds Varki, A. et al.) (Cold Spring Harbor Laboratory Press, 2017)

128. Boisset, J.-C. et al. Mapping the physical network of cellular interactions. Nat. Methods 15, 547-553 (2018).

This work introduces ProximID, a computational approach for building a cell-cell network from physical interactions and single-cell RNA-seq data

129. Shao, X., Lu, X., Liao, J., Chen, H. \& Fan, X New avenues for systematically inferring cell-cell communication: through single-cell transcriptomics 
data. Protein Cell https://doi.org/10.1007/s13238020-00727-5 (2020).

130. Giladi, A. et al. Dissecting cellular crosstalk by sequencing physically interacting cells. Nat. Biotechnol. https://doi.org/10.1038/s41587-020 0442-2 (2020).

\section{This work describes PIC-seq, a transcriptomics} technology to study physically interacting cells.

131. Halpern, K. B. et al. Paired-cell sequencing enables spatial gene expression mapping of liver endothelial cells. Nat. Biotechnol. 36, 962-970 (2018).

132. Szczerba, B. M. et al. Neutrophils escort circulating tumour cells to enable cell cycle progression. Nature 566, 553-557 (2019).

133. Schapiro, D. et al. histoCAT: analysis of cell phenotypes and interactions in multiplex image cytometry data. Nat. Methods 14, 873-876 (2017).

134. Lundberg, E. \& Borner, G. H. H. Spatial proteomics: a powerful discovery tool for cell biology. Nat. Rev. Mol. Cell Biol. 20, 285-302 (2019).

135. Ren, X. et al. Reconstruction of cell spatial organization from single-cell RNA sequencing data based on ligand-receptor mediated self-assembly. Cell Res. https://doi.org/10.1038/s41422-020-0353-2 (2020).

136. Francis, K. \& Palsson, B. O. Effective intercellular communication distances are determined by the relative time constants for cyto/chemokine secretion and diffusion. Proc. Natl Acad. Sci. USA 94, 12258-12262 (1997).

137. Atakan, B. in Molecular Communications and Nanonetworks: From Nature to Practical Systems (ed. Atakan, B.) 105-143 (Springer, 2014).

138. Wagner, D. E. \& Klein, A. M. Lineage tracing meets single-cell omics: opportunities and challenges. Nat. Rev. Genet. 21, 410-427 (2020)

139. Zhang, J., Nie, Q. \& Zhou, T. Revealing dynamic mechanisms of cell fate decisions from single-cell transcriptomic data. Front. Genet. 10, 1280 (2019).

140. Kuchta, K. et al. Predicting proteome dynamics using gene expression data. Sci. Rep. 8, 13866 (2018).

141. Krieglstein, C. F. \& Granger, D. N. Adhesion molecules and their role in vascular disease. Am. J. Hypertens. 14, 44S-54S (2001).

142. Nourani, E., Khunjush, F. \& Durmus, S. Computational approaches for prediction of pathogenhost protein-protein interactions. Front. Microbiol. 6 , 94 (2015).

143. Durmus, , S., Çakır, T., Özgür, A. \& Guthke, R. A review on computational systems biology of pathogen-host interactions. Front. Microbiol. 6, 235 (2015).

144. Schulze, S., Henkel, S. G., Driesch, D., Guthke, R. $\Sigma$ Linde, J. Computational prediction of molecular pathogen-host interactions based on dual transcriptome data. Front. Microbiol. 6, 65 (2015).

145. Mirrashidi, K. M. et al. Global mapping of the Inc-human interactome reveals that retromer restricts chlamydia infection. Cell Host Microbe 18, 109-121 (2015).

146. Thompson, L. R. et al. A communal catalogue reveals Earth's multiscale microbial diversity. Nature $\mathbf{5 5 1}$, 457-463 (2017).

147. McDonald, D. et al. American gut: an open platform for citizen science microbiome research. mSystems 3 , (2018).

148. Kyrpides, N. C., Eloe-Fadrosh, E. A. \& Ivanova, N. N. Microbiome data science: understanding our microbial planet. Trends Microbiol. 24, 425-427 (2016).

149. Gonzalez, A. et al. Qiita: rapid, web-enabled microbiome meta-analysis. Nat. Methods 15 796-798 (2018)
150. Zuñiga, C. et al. Environmental stimuli drive a transition from cooperation to competition in synthetic phototrophic communities. Nat. Microbiol. 4, 2184-2191 (2019).

151. Lapek, J. D. Jr et al. Defining host responses during systemic bacterial infection through construction of a murine organ proteome atlas. Cell Syst. 6, 579-592.e4 (2018).

152. Penaranda, C. \& Hung, D. T. Single-cell RNA sequencing to understand host-pathogen interactions. ACS Infect. Dis. 5, 336-344 (2019).

153. Jäger, S. et al. Global landscape of HIV-human protein complexes. Nature 481, 365-370 (2011).

154. Batra, J. et al. Protein interaction mapping identifies RBBP6 as a negative regulator of ebola virus replication. Cell 175, 1917-1930.e13 (2018).

155. Shah, P. S. et al. Comparative flavivirus-host protein interaction mapping reveals mechanisms of Dengue and Zika virus pathogenesis. Cell 175, 1931-1945.e18 (2018).

156. Gordon, D. E. et al. A SARS-CoV-2 protein interaction map reveals targets for drug repurposing. Nature https://doi.org/10.1038/s41586-020-2286-9 (2020)

157. Adli, M. The CRISPR tool kit for genome editing and beyond. Nat. Commun. 9, 1911 (2018).

158. Hsu, M.-N. et al. CRISPR technologies for stem cell engineering and regenerative medicine. Biotechnol. Adv. 37, 107447 (2019).

159. Kwon, E. D. et al. Manipulation of T cell costimulatory and inhibitory signals for immunotherapy of prostate cancer. Proc. Natl Acad. Sci. USA 94, 8099-8103 (1997).

160. Xu, W., Atkins, M. B. \& McDermott, D. F. Checkpoint inhibitor immunotherapy in kidney cancer. Nat. Rev. Urol. 17, 137-150 (2020).

161. Huang, H. et al. Cell-cell contact-induced gene editing/ activation in mammalian cells using a synNotchCRISPR/Cas9 system. Protein Cell 11, 299-303 (2020).

162. de Juan, D., Pazos, F. \& Valencia, A. Emerging methods in protein co-evolution. Nat. Rev. Genet. 14, 249-261 (2013)

163. Kuhlman, B. \& Bradley, P. Advances in protein structure prediction and design. Nat. Rev. Mol Cell Biol. 20, 681-697 (2019)

164. Chupakhin, V., Marcou, G., Baskin, I., Varnek, A. $\S$ Rognan, D. Predicting ligand binding modes from neural networks trained on protein-ligand interaction fingerprints. J. Chem. Inf. Model. 53, 763-772 (2013).

165. Fenoy, E., Izarzugaza, J. M. G., Jurtz, V., Brunak, S. $\&$ Nielsen, M. A generic deep convolutional neural network framework for prediction of receptor-ligand interactions-NetPhosPan: application to kinase phosphorylation prediction. Bioinformatics 35 1098-1107 (2019).

166. Kveler, K. et al. Immune-centric network of cytokines and cells in disease context identified by computational mining of PubMed. Nat. Biotechnol. 36, 651-659 (2018)

167. Ashburner, M. et al. Gene ontology: tool for the unification of biology. Nat. Genet. 25, 25-29 (2000).

168. UniProt Consortium. UniProt: a hub for protein information. Nucleic Acids Res. 43, D204-D212 (2015).

169. Kanehisa, M. \& Goto, S. KEGG: Kyoto Encyclopedia of Genes and Genomes. Nucleic Acids Res. 28, 27-30 (2000).

170. Waterhouse, R. M., Tegenfeldt, F., Li, J., Zdobnov, E. M \& Kriventseva, E. V. OrthoDB: a hierarchical catalog of animal, fungal and bacterial orthologs. Nucleic Acids Res. 41, D358-D365 (2013).
171. Reimand, J., Kull, M., Peterson, H., Hansen, J. \& Vilo, J. g:profiler - a web-based toolset for functional profiling of gene lists from large-scale experiments. Nucleic Acids Res. 35, W193-W200 (2007).

172. Uhlén, M. et al. Tissue-based map of the human proteome. Science 347, 1260419 (2015).

173. Szklarczyk, D. et al. STRING v11: protein-protein association networks with increased coverage, supporting functional discovery in genome-wide experimental datasets. Nucleic Acids Res. 47, D607-D613 (2019).

174. Stark, C. et al. BioGRID: a general repository for interaction datasets. Nucleic Acids Res. 34, D535-D539 (2006).

175. Gioutlakis, A., Klapa, M. I. \& Moschonas, N. K. PICKLE 2.0: a human protein-protein interaction meta-database employing data integration via genetic information ontology. PLOS ONE 12, e0186039 (2017).

176. Prieto, C. \& De Las Rivas, J. APID: agile protein interaction DataAnalyzer. Nucleic Acids Res. 34, W298-W302 (2006).

177. Hermjakob, H. et al. IntAct: an open source molecular interaction database. Nucleic Acids Res. 32, D452-D455 (2004)

178. Cerami, E. G. et al. Pathway commons, a web resource for biological pathway data. Nucleic Acids Res. 39, D685-D690 (2011).

179. Pawson, A. J. et al. The IUPHAR/BPS Guide to PHARMACOLOGY: an expert-driven knowledgebase of drug targets and their ligands. Nucleic Acids Res. 42, D1098-D1106 (2014).

Acknowledgements

E.A. is supported by the Chilean Agencia Nacional de Investigaciōn y Desarrollo through its scholarship programme DOCTORADO BECAS CHILE/2018 - 72190270 and by the Fulbright Commission. A.O. is supported by the US NLM (T15LM011271). O.H. is supported by the US NIH (U01CA196406). The authors also thank A. Perez-Lopez, C. Zuñiga, J. Tibocha-Bonilla, L. Zaramela, M. Kumar and P. Tamayo for providing meaningful feedback and discussion. This work was also supported by the US NIGMS (R35 GM119850; N.E.L.).

\section{Author contributions}

E.A. and A.O. researched the literature. All authors contributed to discussions of the content and wrote, reviewed and/or edited the manuscript before submission.

\section{Competing interests}

The authors declare no competing interests.

Peer review information

Nature Reviews Genetics thanks O. Nie; V. Soumelis; and R. Vento-Tormo, who co-reviewed with A. Arutyunyan, for their contribution to the peer review of this work.

Publisher's note

Springer Nature remains neutral with regard to jurisdictional claims in published maps and institutional affiliations.

\section{Supplementary information}

Supplementary information is available for this paper at https://doi.org/10.1038/s41576-020-00292-x.

\section{RELATED LINKS}

Ligand-receptor pair repository: https://github.com/

LewisLabUCSD/Ligand-Receptor-Pairs

(c) Springer Nature Limited 2020 